\title{
Discriminative Block-Diagonal Representation Learning for Image Recognition
}

\author{
Zheng Zhang, Yong Xu, Senior Member, IEEE, Ling Shao, Senior Member, IEEE, and Jian Yang
}

\begin{abstract}
Existing block-diagonal representation studies mainly focuses on casting block-diagonal regularization on training data, while only little attention is dedicated to concurrently learning both block-diagonal representations of training and test data. In this paper, we propose a discriminative block-diagonal low-rank representation (BDLRR) method for recognition. In particular, the elaborate BDLRR is formulated as a joint optimization problem of shrinking the unfavorable representation from off-block-diagonal elements and strengthening the compact block-diagonal representation under the semisupervised framework of LRR. To this end, we first impose penalty constraints on the negative representation to eliminate the correlation between different classes such that the incoherence criterion of the extraclass representation is boosted. Moreover, a constructed subspace model is developed to enhance the self-expressive power of training samples and further build the representation bridge between the training and test samples, such that the coherence of the learned intraclass representation is consistently heightened. Finally, the resulting optimization problem is solved elegantly by employing an alternative optimization strategy, and a simple recognition algorithm on the learned representation is utilized for final prediction. Extensive experimental results demonstrate that the proposed method achieves superb recognition results on four face image data sets, three character data sets, and the 15 scene multicategories data set. It not only shows superior potential on image recognition but also outperforms the state-of-the-art methods.
\end{abstract}

Index Terms- Block-diagonal structure, discriminative representation, image recognition, low-rank representation (LRR), sparse representation.

\section{INTRODUCTION}

D ISCRIMINATIVE and effective data representations lay an indispensable role in computer vision and machine learning, because they tremendously influence the performance of various learning systems [1]. A favorable data representation can greatly uncover the underlying information of observed data and intensely facilitate the machine learning methods [2]. As a typical data representation method, sparse representation has earned its high reputation in both theoretical

This work was supported in part by the National Natural Science Foundation of China under Grant 61233011, and in part by the Guangdong Province High-Level Personnel of Special Support Program under Grant 2016TX03X164. (Corresponding author: Yong Xu.)

Z. Zhang and Y.Xu are with the Bio-Computing Research Center, Shenzhen Graduate School, Harbin Institute of Technology, Shenzhen 518055, China (email: darrenzz219@gmail.com; yongxu@ymail.com).

L. Shao is with the School of Computing Sciences, University of East Anglia, Norwich NR4 7TJ, U.K. (e-mail: ling.shao@ieee.org).

J. Yang is with the College of Computer Science and Technology, Nanjing University of Science and Technology, Nanjing 210094, China (e-mail: csjyang@njust.edu.cn).

Color versions of one or more of the figures in this paper are available online at http://ieeexplore.ieee.org. research and practical applications [2]-[4]. Recently, lowrank representation (LRR) has captured considerable attention [5]-[7], and has also been proved to be a powerful solution to a wide range of applications, especially in subspace segmentation [6], feature extraction [7], and image classification [8]-[10]. In this paper, we focus on learning an appropriate data representation by constructing a block-diagonal LRR (BDLRR) for image recognition.

Sparse representation has been widely studied and applied in signal processing, machine learning, and computer vision [3], [11]. The key idea of sparse representation is based on the assumption that each signal can be approximately represented by a linear combination of a few atoms of an overcompleted dictionary. With the successful application of sparse representation-based classification (SRC) [3] in face recognition, numerous SRC-based modifications have been proposed. For example, Nie et al. [13] introduced an efficient and robust feature selection method by imposing the $l_{21}$-norm constraint on both loss function and regularization terms. $\mathrm{Xu}$ et al. [14] proposed the semisupervised sparse representation by employing a coarse-to-fine strategy, and Lu et al. [15] developed a weighted sparse representationbased classifier by leveraging both data locality and linearity to sparse coding. Based on the basic theorem [4] that locality can always lead to sparsity but not necessarily vice versa, the locality-constrained linear coding (LLC) [4] method achieves the sparse target by enforcing the locality embedding of codebook. In addition, some researchers argue that sparsity is not the ultimate reason of achieving decent recognition results [12], [16]-[18]. For example, Zhang et al. [16] presented a collaborative representationbased classification (CRC) method by employing the $l_{2}$ norm regularization rather than $l_{1}$-norm regularization for face recognition. It is demonstrated that CRC can achieve comparable performance but more efficient than SRC [16]. The linear regression-based classifica- tion (LRC) [18] is another well-known representation-based method. More specifically, LRC exploits each class of training samples to represent the test sample, and classifies it to the class leading to the minimum representation residual. A recent survey [2] comprehensively reviews most representative sparse representation-based algorithms, and empirically summarizes its wide applications from both theoretical and practical perspectives.

Recently, LRR has gained increasing interest from different research fields. It is noted that the sparsity constraint can only dominate the local structure of each data vector, whereas the low-rank constraint can directly control the global structure of 
data [19]. Furthermore, LRR can greatly capture the underlying correlation behind the observed data [19], [20]. The most representative low-rank method, robust principal component analysis (RPCA) [21], was proposed to recover the clean data with the low-rank constraint from corrupted observations. In particular, RPCA first assumes that the observations lie in a single subspace such that they can be decomposed into two separate components, i.e., the low-rank and sparse noise parts. However, RPCA cannot handle the situation where corrupted or outlying data are drawn from a union of multiple subspaces. To this end, Liu et al. [6] proposed to perform matrix recovery by exploiting LRR for subspace segmentation. The latent LRR (LatLRR) [7] was then developed for joint subspace segmentation and feature extraction by discovering the hidden information underlying the observations. Moreover, lots of LRR-based dictionary learning methods were proposed for robust image classification. For example, Zhang et al. [10] constructed a structured LRR (SLRR) by regularizing all training images of the same class to have the same representation code. However, the ideal structure in SLRR is questionable, because, though data from the same class usually lie in the same subspace, it does not mean that images belonging to the same class should have the same data representation. Wei et al. [22] developed a low-rank matrix approximation method by learning subdictionaries independently for each class and meanwhile enforcing the structural incoherence between different classes. Li et al. [23] explored a classwise block-diagonal structure (CBDS) dictionary learning method, which learned discriminative LRR by imposing the classwise structure constraint. In addition, some variations of LRRbased methods were proposed to solve different prob- lems. For example, Li et al. [24] designed a cross-view low-rank analysis framework to address the multiview outlier detection problem. Zhuang et al. [25] presented a nonnegative low-rank sparse graph construction method for semisupervised learning. As a result, it is widely agreed that the low-rank cri- terion indeed can disclose the potential data structures of dif-ferent classes or tasks' correlation patterns, such that the effectiveness of the learned data representation can be enhanced.

There is a well-attested fact that the sparse representation in [3] is a discriminative representation, whereas it only considers the data representation of each input signal independently, which does not take advantage of the global structural information in the set. In addition, existing research [22]-[24] has demonstrated that imposing specific structures on the LRR matrix is beneficial to improve the discriminative capability of data representation. However, the performance of these methods is still far from being satisfactory. The main reason may be that these methods cannot perfectly transfer the original data features to the discriminative feature representations. Based on the well-explored self-expression property [26], the ideal block-diagonal representation can capture the underlying data information of samples by embedding the global semantic structure information and discriminative identification capability [10]. Consequently, promising results can be achieved if the discriminative data representation with the block-diagonal structure is exploited for recognition. In this paper, a novel BDLRR method is proposed to learn discriminative data representations, which can simultaneously shrink the off-block-diagonal components and highlight the block-diagonal representation under the framework of LRR. More specifically, BDLRR first eliminates the negative representation and boosts the incoherence of the extra-class representation by minimizing the offblock-diagonal representation, such that it can remove the noisy representation and transfer the positive representa- tion to the block-diagonal components. Furthermore, BDLRR constructs a subspace model to enhance the self-expressive power of training samples and simultaneously bridge the representation gap between the training and test samples in a semisupervised manner, such that the coherence of the intraclass representation is further improved and the learned representations are consistent. Finally, we introduce an effective iterative algorithm to solve the resulting optimization problem, and our method is evaluated to verify its adaptive capabilities for different recognition tasks. In summary, our key contributions are summarized as follows.

1) A discriminative block-diagonal data representation structure is designed to boost the incoherent power of the extra-class representation by jointly removing the negative representation from the off-block-diagonal components and conveying the positive representation to the block-diagonal structure, such that better discriminative data representations are obtained for recognition tasks.

2) A constructed subspace structure is developed to enhance the coherence of the intraclass representation by simultaneously improving the self-expressive capabilities of training samples and further narrowing the representation gap between training and test samples. Moreover, a low-rank criterion is enforced to capture the underlying feature structures of different classes or tasks' correlation patterns such that more competent representation results are achieved.

3) By virtue of the semisupervised learning superiority, the well-defined representation learning framework simultaneously learns both of discriminative training and test representations to keep consistence of the learned representations for recognition. To accommodate our method for large-scale problems, the out-of-sample extension is further explored to deal with new data instances.

4) An effective optimization strategy based on the alternating direction method of multipliers (ADMM) is developed to solve the resulting optimization problem, and the convergence analysis of the designed optimization problem is presented from both theoretical and experimental perspectives.

The rest of this paper is organized as follows. We briefly review the related work on the low-rank theory in Section II. Then, we elaborate the proposed BDLRR method in Section III, and the solution to the optimization problem of the proposed BDLRR method is presented in Section IV. Section V reports extensive experimental results, as well as convergence and parameter sensitiveness analysis. Finally, the conclusion remarks are given in Section VI.

\section{RELATED WORK}

In this section, we give a brief review of two typical lowrank criterion-based methods, i.e., LRR [6] and RPCA [21]. 
Let us first introduce our notations used in this paper. Matrices are represented with bold uppercase letters, e.g., $\boldsymbol{X}$, and column vectors are denoted by bold lower letters, e.g., $\boldsymbol{x}$. In particular, $\boldsymbol{I}$ denotes an identity matrix, and the entries of a matrix or vector are denoted by using [.] with subscripts. The $i$ th row and $j$ th column element of matrix $\boldsymbol{X}$ is denoted as $\boldsymbol{x}_{i j}$, and the block-diagonal matrix composed of a collection of matrices $\left[\boldsymbol{X}_{1}, \ldots, \boldsymbol{X}_{C}\right]$ is denoted by

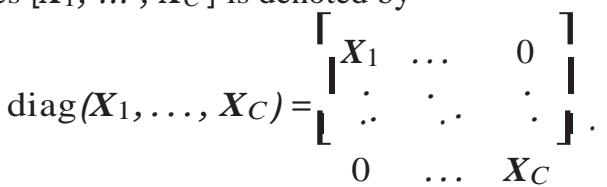

A matrix's $l_{0}, l_{1}$, and $l_{21}$ norms are denoted as $\times \boldsymbol{X} \times \times_{0}=$ $\#\left\{(i, j): \boldsymbol{x}_{i j} *=0\right\}, \times \boldsymbol{X} \times \times_{1}=-_{i j}\left|\boldsymbol{x}_{i j}\right|$, and $\times \boldsymbol{X} \times 21=$ ${ }_{j} \times \boldsymbol{X}_{. j} \times$, respectively. The norm induced by the $l_{\infty}$-norm on the matrix is denoted as $\times X \times_{\infty}=\max _{i}{ }_{j}\left|\boldsymbol{x}_{i j}\right|$. The matrix Frobenius norm designates $\times \boldsymbol{X}_{\mathrm{x}_{F}}^{2}=\operatorname{tr}\left(\boldsymbol{X}^{T} \boldsymbol{X}\right)=\operatorname{tr}$ $\left(\boldsymbol{X} \boldsymbol{X}^{T}\right)={ }_{i j} \boldsymbol{x}^{2}{ }_{i j}$ where $\operatorname{tr}(\cdot)$ is the trace operator. $\times \boldsymbol{X}_{*}$ is the trace or nuclear norm of matrix $\boldsymbol{X}$, i.e., $\times \boldsymbol{X} \mathrm{x}_{*}={ }^{-}\left|\sigma_{i}\right|$, where $\sigma_{i}$ is the $i$ th singular value of matrix $\boldsymbol{X} . \boldsymbol{X}^{T}$ denotes the thensposed matrix of $X_{k} 0_{m y}$ denotes an all-zerp matrix ${ }_{1}{ }^{T \text { with }}$

$$
\mathrm{s}^{-},-\mathrm{s}
$$

\section{A. Robust Principal Component Analysis}

Suppose that $\boldsymbol{X}=\left[\boldsymbol{x}_{1}, \ldots, \boldsymbol{x}_{n}\right] \in \mathrm{R}^{d \times n}$ is the observed data matrix and composed of $n$ samples, where each column is a sample vector and usually has been highly corrupted. The main objective of RPCA is to determine a low-rank matrix $\boldsymbol{X}_{0}$ from the corrupted observations $\boldsymbol{X}$, and, meanwhile, filter out the sparse noise components $\boldsymbol{E}$, i.e., $\boldsymbol{X}=\boldsymbol{X}_{0}+\boldsymbol{E}$. Consequently, the objective function of RPCA can be easily formulated as

$$
\min _{\boldsymbol{X}_{0}, \boldsymbol{E}} \operatorname{rank}\left(\boldsymbol{X}_{0}\right)+\lambda \times \boldsymbol{E} \times_{0} \text { s.t. } \boldsymbol{X}=\boldsymbol{X}_{0}+\boldsymbol{E}
$$

where the $\operatorname{rank}(\cdot)$ operator denotes the rank of matrix $\boldsymbol{X}_{0}, \lambda$ is the balance parameter, and $\times \cdot \times_{0}$ means the $l_{0}$ pseudonorm. Given an appropriate value of $\lambda$, RPCA can recover the clean data by $\boldsymbol{X}_{0}$. Due to the discrete properties of the rank function and the $l_{0}$-norm minimization, both of them are NPhard problems and even difficult to approximate. An advis-

able choice [21] is to replace the rank constraint and $l_{0}$-norm regularization by the nuclear norm and $l_{1}$-norm regularization, respectively. As a result, problem (1) can be reformulated as

$$
\min _{\boldsymbol{X}_{0}, \boldsymbol{E}} \times \boldsymbol{X}_{0} \times{ }_{*}+\lambda \times \boldsymbol{E} \times{ }_{1} \text { s.t. } \boldsymbol{X}=\boldsymbol{X}_{0}+\boldsymbol{E}
$$

where $x \cdot x_{*}$ and $x \cdot x_{1}$ are the nuclear norm and $l_{1}$-norm, respectively. It is known that problem (2) can be efficiently solved by the augmented Lagrange multiplier (ALM) method [27].

\section{B. Low-Rank Representation-Based Method}

It is noted that RPCA is essentially based on the priori hypothesis that the observed data is approximately drawn from a low-rank subspace, that is, data can be described by a single subspace [6]. However, this assumption is very difficult to be satisfied for real-world data sets, where multiple subspaces are more reasonable. To this end, LRR [6] assumes that each data can be approximately represented by a union of several linear low-rank subspaces. The objective function of LRR is formulated as

$$
\min _{\boldsymbol{Z}, \boldsymbol{E}} \operatorname{rank}(\boldsymbol{Z})+\lambda \times \boldsymbol{E} \times \times_{l} \text { s.t. } \boldsymbol{X}=\boldsymbol{D Z}+\boldsymbol{E}
$$

where $\boldsymbol{D}$ and $\lambda$ are the dictionary and balance parameter, respectively. $\times \cdot \times_{l}$ indicates the constraint of different norms, and imposing different norms tends to remove specific noise as illustrated in [6]. For example, the matrix Frobenius norm can effectively capture Gaussian noise, while the $l_{1}$-norm can better process the random noise or corruptions. Similar to RPCA, problem (3) can be approximately reformulated as

$$
\min _{\boldsymbol{Z}, \boldsymbol{E}} \times \boldsymbol{Z} \times{ }_{*}+\lambda \times \boldsymbol{E} \times l \text { s.t. } \boldsymbol{X}=\boldsymbol{D Z}+\boldsymbol{E}
$$

which can also be effectively solved by the ALM algorithm [6], [27].

\section{$\mathrm{III}_{\text {L PRQPOSED }}$ BLOCK-DIAGONAL
OW ANK EPRESENTATION}

In this section, we introduce a novel BDLRR method, which collaboratively learns appropriate block-diagonal representations of training and test samples by jointly enforcing the incoherence of extra-class data representations and enhancing

the coherence of intraclass data representations.

Assumption 1: Let $\boldsymbol{X}=\left[\boldsymbol{X}_{1}, \ldots, \boldsymbol{X}_{C}\right] \in \mathrm{R}^{d \times n}$ denote $N$ training samples with a dimension of $d$ from $C$ classes, where each column is a sample vector. Suppose that all the samples are rearranged based on the class labels, and each class of training samples is stacked together to form a submatrix $\boldsymbol{X}_{i} \in \mathrm{R}^{d \times n_{i}}$, which denotes $n_{i}$ samples from the $i$ th class $(i=1,2, \ldots, C)$.

Definition 1 (Self-Expressiveness Property [26]): Each data instance from a union of multiple subspaces can be effectively represented by a linear combination of other data instances, which is referred as the self-expressiveness property.

Definition 2: Suppose that a data point $\boldsymbol{y} \in \mathrm{R}^{d}$ is from the $i$ th class. $\boldsymbol{z}$ is a solution of the linear equation $\boldsymbol{y}=\boldsymbol{X} \boldsymbol{z}$, where the subvectors $z_{j}(j=1,2, \ldots, C)$ of $z$, respectively, correspond to the $j$ th class. Based on the self-expressiveness property, the submatrix $\boldsymbol{X}_{i}$ should be able to well represent $\boldsymbol{y}$, and there is $\boldsymbol{y} \approx \boldsymbol{X}_{i} \boldsymbol{z}_{i}$. We define $\boldsymbol{z}_{i}$ as the intraclass representation, otherwise, the coding coefficients $z_{j}(j *=i$ ) are called the extra-class representation.

It is worth noting that the self-expressiveness property has already been successfully utilized in the context of classification [3], [22], low-rank matrix approximation [6] and clustering [26]. Typically, SSC and LRR are the most representative methods, and the explicit self-expressiveness formulation, $\boldsymbol{X}=$ $\boldsymbol{X} \boldsymbol{Z}$, is easily satisfied, where $\boldsymbol{Z}$ is data representation. Furthermore, in the presence of the self-expressiveness property, the key underlying observation of SSC and LRR is disclosed that each data point in a data set can be ideally represented by a linear combination of a few points from its own subspace. Based on this observation and Assumption 1, the desired selfexpressive representation should be block-diagonal and the 
obtained data representation is sufficiently discriminative. So, the ideal block-diagonal structure-based representation is

$$
\boldsymbol{X}=\boldsymbol{X} \hat{\boldsymbol{Z}} \text { s.t. } \hat{\boldsymbol{Z}}=\operatorname{diag}(\boldsymbol{Z})
$$

where $\boldsymbol{Z}=\left[\boldsymbol{Z}_{11}, \ldots, \boldsymbol{Z}_{C C}\right]$, and $\boldsymbol{Z}_{i j}$ is the representation coefficient of $\boldsymbol{X}_{i}$ corresponding to $\boldsymbol{X}_{j}$. However, the absolute block-diagonal structure is not easy to learn. To this end, it is natural to assume that the off-block-diagonal components are as small as possible to enhance the incoherent extra-class representation, which means that $\boldsymbol{Z}_{i j}$ tends to a zero submatrix for $i *=j$. In addition, the coherent intraclass representation at

the same time is further boosted. We formulate the following structured representation as:

$$
\left.\min _{\boldsymbol{Z}} \lambda_{1} \times \boldsymbol{A} \mathrm{S}\right) \mathrm{Z}_{F}^{2}+\lambda_{2} \times \boldsymbol{D}(\mathrm{S}) \boldsymbol{Z} \times{ }_{0} \text { s.t. } \boldsymbol{X}=\boldsymbol{X} \boldsymbol{Z}
$$

where $\lambda_{1}$ and $\lambda_{2}$ are positive constants to weigh corresponding terms, (\$) indicates the Hadamard product (i.e., elementwise product), and $\boldsymbol{X} \in \mathrm{R}^{d \times n}$. More specifically, the first term attempts to minimize the off-block-diagonat entries, and

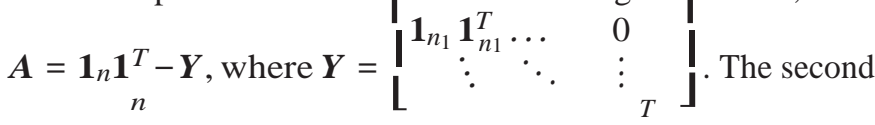
$0 \quad \ldots \mathbf{1}_{n_{C}} \mathbf{1}_{n_{C}}$

term is the constructed subspace measure to improve the coherent representation of intraclass representation. $\boldsymbol{d}_{i j}$ is a distance metric between $\boldsymbol{x}_{i}$ and $\boldsymbol{x}_{j}$ such that similar samples have high probabilities to be similar data representations. There are many distance metric methods. In this paper, we simply define the distance between two samples as the square of the Euclidean distance, i.e., $\times \boldsymbol{x}_{i}-\boldsymbol{x}_{j}{ }^{2}$. Because solving the $l_{0^{-}}$ norm minimization problem is an ${ }^{2}$ NP-hard problem, a relaxed counterpart of the second term is formulated as

$\times \boldsymbol{D}\left(\mathrm{S} Z \times{ }_{1}\right.$. Thus, problem (6) can be reformulated as

$$
\min _{\boldsymbol{Z}} \lambda_{1} \times \boldsymbol{A}\left(\mathrm{S} \boldsymbol{Z} \times_{F}{ }_{F}+\lambda_{2} \times \boldsymbol{D}(\mathrm{S}) \boldsymbol{Z} \times{ }_{1} \text { s.t. } \boldsymbol{X}=\boldsymbol{X} \boldsymbol{Z}\right. \text {. }
$$

In general, a low-rank criterion can further capture the underlying classes' correlation patterns such that the performance of resulting models can be improved [6], [10], [20]. By integrating problems (7) and (4), we propose the following objective function for the semisupervised BDLRR:

$$
\begin{aligned}
& \min _{\boldsymbol{Z}, \boldsymbol{E}} \times \boldsymbol{Z} \times \times_{*}+\lambda_{1} \times \tilde{\boldsymbol{A}} \mathbf{S} \times_{F}^{2}+\lambda_{2} \times \boldsymbol{D} \mathbf{S} \times_{1}+\lambda_{3} \times \boldsymbol{E} \times 21 \\
& \text { s.t. } \boldsymbol{X}=\boldsymbol{X}_{\mathrm{tr}} \boldsymbol{Z}+\boldsymbol{E}
\end{aligned}
$$

where $\lambda_{1}, \lambda_{2}$, and $\lambda_{3}$ are positive scalars that weigh the corresponding terms in (8) $\boldsymbol{X}_{\text {tr }} \in \mathrm{R}^{d \times n}$ is the training data matrix and $\boldsymbol{X} \in \mathrm{R}^{d \times N}$ includes both training and test samples, i.e., $\boldsymbol{X}=\left[\boldsymbol{X}_{\mathbf{t r}}, \boldsymbol{X}_{\mathrm{tt}}\right]$. For the second term, $\tilde{\boldsymbol{A}}=\left[\boldsymbol{A}, \mathbf{1}_{n} \mathbf{1}_{N-n}^{T}\right]$, where $\boldsymbol{A}$ is the same as (6), and data representation $\boldsymbol{Z}=\left[\boldsymbol{Z}_{\mathrm{tr}}, \boldsymbol{Z}_{\mathrm{tt}}\right]$ such that an implicit $\times \boldsymbol{Z}_{\mathrm{tt}} \times{ }_{F}$ term ${ }_{n \times N}$ is imposed to avoid overfitting. For the third term, $\boldsymbol{D} \in$ $\mathrm{R} \quad$ is denoted as the distance between training samples $\boldsymbol{X}_{\mathrm{tr}}$ and all samples $\boldsymbol{X}$ such that the coherent representation of both $\boldsymbol{X}_{\mathrm{tr}}$ and $\boldsymbol{X}_{\mathrm{tt}}$ corresponding to $\boldsymbol{X}_{\mathrm{tr}}$ can be enhanced simultaneously. $\boldsymbol{E}$ denotes the noise term with the $l_{21}$-norm regularization to capture sample-specific noise information [6]. Moreover, data representation $\boldsymbol{Z}$ of training and test samples is incorporated into a unified optimization problem such that

$\boldsymbol{Z}_{\mathrm{tr}}$ and $\boldsymbol{Z}_{\mathrm{tt}}$ are both optimal and discriminative.

\section{OptimizATION AND ALGORITHM ANALYSis}

To solve the optimization problem of BDLRR in (8), we propose to utilize an alternating direction method, and separate the problem into several subproblems, which have closed-form solutions.

\section{A. Optimization Algorithm}

To solve optimization problem (8), we first make an equivalent transformation by introducing two auxiliary variables to make the problem separable, and then, problem (8) can be

rewritten as

$$
\begin{aligned}
& \min _{\boldsymbol{P}, \boldsymbol{Z}, \boldsymbol{Q}, \boldsymbol{E}} \times \boldsymbol{P} \times{ }_{*}+\frac{\lambda_{1}}{2} \times \tilde{\boldsymbol{A}} \mathbf{S} \boldsymbol{Z} \times{ }_{F}^{2}+\lambda_{2} \times \boldsymbol{D} \mathbf{S} \boldsymbol{Q} \times{ }_{1}+\lambda_{3} \times \boldsymbol{E} \times 21 \\
& \text { s.t. } \boldsymbol{X}=\boldsymbol{X}_{\mathrm{tr}} \boldsymbol{Z}+\boldsymbol{E}, \boldsymbol{P}=\boldsymbol{Z}, \boldsymbol{Q}=\boldsymbol{Z}
\end{aligned}
$$

Then, we can get the following objective function of the problem by the ALM method. Here, the augmented Lagrangian function of problem (9) is

$$
\begin{aligned}
& L\left(P, Z, Q, \underset{\lambda_{1}}{E}, C_{1}, C_{2}, C_{3}\right) \\
& =\times \boldsymbol{P} \times_{*}+\overline{-} \times \tilde{\boldsymbol{A}} \mathbf{S Z} \times_{F} \\
& +\lambda_{2} \times \boldsymbol{D}\left(\mathrm{S} \boldsymbol{Q} \times{ }_{1}+\lambda_{3} \times \boldsymbol{E} \times{ }_{21}+\left(\boldsymbol{C}_{1}, \boldsymbol{X}-\boldsymbol{X}_{\mathrm{tr}} \boldsymbol{Z}-\boldsymbol{E}\right)\right. \\
& \begin{array}{l}
+.\left(C_{2}, \boldsymbol{P}-\underset{Z}{Z}\right)+\left(\boldsymbol{C}_{3}, \boldsymbol{Q}-\boldsymbol{Z}\right)+\frac{\mu}{2^{2}} \\
\times \times \boldsymbol{P}-\boldsymbol{Z} \times{ }_{F}+\times \boldsymbol{X}-\boldsymbol{X}_{\mathrm{tr}} \boldsymbol{Z}-\boldsymbol{E} \times{ }_{F}+\times \boldsymbol{Q}-\boldsymbol{Z} \times{ }_{F}^{?}
\end{array}
\end{aligned}
$$

where $(\boldsymbol{P}, \boldsymbol{Q})=\operatorname{tr}\left(\boldsymbol{P}^{T} \boldsymbol{Q}\right) . \boldsymbol{C}_{1}, \boldsymbol{C}_{2}$, and $\boldsymbol{C}_{3}$ are the Lagrangian multipliers, and $\mu>0$ is a penalty parameter. The augmented Lagrangian is minimized along one coordinate direction at each iteration, i.e., minimizing the loss with respect to one variable with the remaining variables fixed.

We introduce the detailed procedures as follows.

1) Updating $\boldsymbol{Z}$ : Fix the other variables and update $\boldsymbol{Z}$ by solving the following problem:

$$
\begin{aligned}
& L=\min _{\boldsymbol{Z}}{\frac{\lambda_{1}}{\times}}^{\tilde{\boldsymbol{A}}} \text { (S) } \boldsymbol{Z}_{\times_{F}}^{2}+\left(\boldsymbol{C}_{1}^{t}, \boldsymbol{X}-\boldsymbol{X}_{\operatorname{tr}}^{\boldsymbol{Z}}-\boldsymbol{E}^{t}\right) \\
& +\left(\boldsymbol{C}_{2}^{t}, \boldsymbol{P}^{t+1}-\boldsymbol{Z}\right)+\left(\boldsymbol{C}_{3}^{t}, \boldsymbol{Q}^{t}-\boldsymbol{Z}\right) \\
& +\frac{\mu^{t}}{2} \times \boldsymbol{P}^{t+1}-\boldsymbol{Z}_{F}^{2}+\times \boldsymbol{X}-\boldsymbol{X}_{\mathrm{tr}} \boldsymbol{Z}-\boldsymbol{E}{ }^{\mathrm{t}}{ }^{2}{ }_{F}+\times \boldsymbol{Q}^{t}-\boldsymbol{Z} \times_{F}^{2} . \\
& =\frac{\lambda_{1}}{2} \times \tilde{A} \mathrm{SZ}_{F}^{2}+\frac{\mu^{t}}{2} \\
& \times^{-} \times \boldsymbol{X}-\boldsymbol{X}_{\mathrm{tr}} \boldsymbol{Z}-\boldsymbol{E}^{t}+\frac{\boldsymbol{C}}{\boldsymbol{t}}_{\times_{F}}^{2}+\times \boldsymbol{P}_{t+1}
\end{aligned}
$$

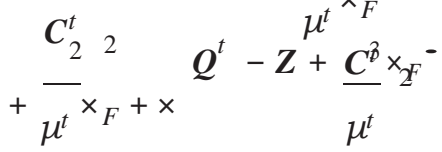


which is equivalent to

$$
\begin{aligned}
& L=\min \frac{\lambda_{1}}{2} \times \boldsymbol{Z}-\boldsymbol{R} \times_{F}^{2} \\
& +{ }_{2}^{\boldsymbol{\mu t}^{t^{-}}}{ }_{2}^{2} \boldsymbol{X}-\boldsymbol{X}_{\mathrm{tr}} \boldsymbol{Z}-\boldsymbol{E}^{t}+{\frac{\boldsymbol{C}_{1}^{t}}{\mu^{t}}}^{2}+\boldsymbol{P}^{t+1}-\boldsymbol{Z}+\frac{\boldsymbol{C}_{2}^{t}}{\mu_{t}}{ }^{2} \\
& +Q^{t}-Z+{\frac{C_{\frac{3}{3}}}{u^{t}}}_{F}^{\mu^{t}}{ }^{2}-
\end{aligned}
$$

where $\boldsymbol{R}=\left[\boldsymbol{Y}, \mathbf{0}_{n(N-n)}\right]$ S $\boldsymbol{Z}^{t}$. By setting the derivation $(\partial L / \partial \boldsymbol{Z})=\mathbf{0}$, we can easily infer the optimal solution of $\boldsymbol{Z}$, and the closed-form solution to problem (12) is given by the following form:

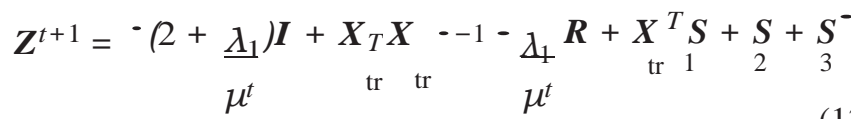

where $\boldsymbol{S}_{1}=\boldsymbol{X}-\boldsymbol{E}^{t}+\left(\boldsymbol{C}^{t} / \mu^{t}\right), \boldsymbol{S}_{2}=\boldsymbol{P}^{t+1}+\left(\boldsymbol{C}^{t} / \mu_{2}^{t}\right)$, and $\boldsymbol{S}_{3}=\boldsymbol{Q}^{t}+\left(\boldsymbol{C}^{t}\left\langle\mu^{t}\right)\right.$.

2) Updating $\boldsymbol{P}$ : When fixing the other variables, the objective function of (10) is degenerated into a function with respect to $\boldsymbol{P}$, that is

$$
\begin{aligned}
& \left.\boldsymbol{P}^{t+1}=\underset{\boldsymbol{P}}{\arg \min } \times \underset{*}{\boldsymbol{P} \times}+\underset{2}{t}, \boldsymbol{P}-\boldsymbol{Z}^{t}\right)+\underset{\underset{\times}{2}}{\mu} \boldsymbol{P}-\boldsymbol{Z}^{t} \times \underset{F}{2}
\end{aligned}
$$

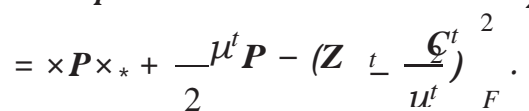

This problem has a closed-form solution by using the singular value thresholding operator [27], [28], that is

$$
\boldsymbol{P}^{t+1}=T_{\frac{1}{\mu^{t}}}{ }^{-} \boldsymbol{Z}^{t}-{\frac{\boldsymbol{C}^{\mathbb{Z}}}{\mu^{t}}}^{-}=\boldsymbol{U S}_{\frac{1}{\mu^{t}}}(\boldsymbol{X}) \boldsymbol{V}^{T}
$$

where $\boldsymbol{U} \boldsymbol{X} \boldsymbol{V}^{T}$ is the singular value decomposition (SVD) of $\left(\boldsymbol{Z}^{t}-\left(\boldsymbol{C}_{2}^{t} / \mu^{t}\right)\right)$, and $\boldsymbol{S}_{\left(1 / \mu^{t}\right)(\cdot)}$ is the soft-thresholding operator [2], [27], which is defined as

$$
S_{\lambda}(x)= \begin{cases}x-\lambda, & \text { if } x>\lambda \\ x+\lambda, & \text { if } x<-\lambda \\ 0, & \text { otherwise. }\end{cases}
$$

the bbjective optinization problem other) withiables are fixed, is degenerated to the following problem:

$$
\begin{aligned}
& L=\min _{\boldsymbol{Q}} \lambda_{2} \times \boldsymbol{D}\left(\mathbf{S} \times \times_{1}+\left(\boldsymbol{C}_{3}^{t}, \boldsymbol{Q}-\boldsymbol{Z}^{t+1}\right)+\frac{\mu_{t}}{2}{ }^{\times} \boldsymbol{Q}_{2}-\boldsymbol{Z}^{t+1} \times_{F}^{2}\right. \\
& =\lambda_{2} \times \boldsymbol{D} S Q \times{ }_{1}+\frac{-}{2}^{\mu^{t}} \boldsymbol{Q}-\boldsymbol{Z}^{+{ }^{+}}-{\frac{\boldsymbol{C}_{3}^{t}}{u^{t}}}_{F}{ }_{F}
\end{aligned}
$$

which can be updated by the elementwise strategy. Obviously, problem (17) can be equivalently decoupled into $n \times N$ subproblems. For the $i$ th row and $j$ th column element $\boldsymbol{Q}_{i j}$, the optimal solution of problem (17) is

$$
\begin{aligned}
\boldsymbol{Q}_{i j}^{t+1} & =\arg \min _{\boldsymbol{Q}_{i j}} \lambda_{2} \boldsymbol{D}_{i j}\left|\boldsymbol{Q}_{i j}\right|+\overline{{ }^{t}} \\
& =\boldsymbol{S}_{\frac{\lambda_{2} \boldsymbol{D}_{i j}}{\mu^{t}}}\left(\boldsymbol{M}_{i j}\right)
\end{aligned}
$$

where $\boldsymbol{M}_{i j}=\boldsymbol{Z}_{i j}^{t+1}-\left(\left(\boldsymbol{C}^{t}\right)_{i j} / \mu^{t}\right)$.
4) Updating $\boldsymbol{E}$ : When other variables are fixed, problem (10) can be converted into the following problem: $\min \lambda_{3} \times \boldsymbol{E} \times 21+\left(\boldsymbol{C}^{t}, \boldsymbol{X}-\boldsymbol{X}_{\mathrm{tr}} \boldsymbol{Z}^{t+1}-\boldsymbol{E}\right)$

$$
\text { E }
$$$$
+\frac{\mu^{t}}{2} \times \boldsymbol{X}-\boldsymbol{X}_{\mathrm{tr}} \boldsymbol{Z}_{t+1}-\boldsymbol{E} \times \underset{F}{2}
$$

which is equivalent to

$$
\min _{\boldsymbol{E}} \lambda_{3} \times \boldsymbol{E} \times 21+\frac{\mu^{t}}{2} \boldsymbol{E}-{ }^{-} \boldsymbol{X}-\boldsymbol{X}_{\mathrm{tr}} \boldsymbol{Z}^{t+1}+\frac{\boldsymbol{C}_{1}^{t}}{\mu^{t}} \quad{ }_{F}^{2} .
$$

The solution to problem (20) is demonstrated in [13]. In particular, let $\boldsymbol{F}=\boldsymbol{X}-\boldsymbol{X}_{\mathrm{tr}} \boldsymbol{Z}^{t+1}+\left(\boldsymbol{C}^{t}\left\{\mu^{t}\right)\right.$, the $i$ th row of $\boldsymbol{E}^{t+1}$ is

$$
\boldsymbol{E}^{t+1}(i,:)= \begin{cases}\frac{\times \boldsymbol{F} \times 2-\overline{\mu^{t}}}{\times \boldsymbol{F}^{i} \times 2} \boldsymbol{F}^{i}, & \text { if } \times \boldsymbol{F}^{i} \times{ }_{2}>\frac{\lambda_{3}}{\lambda_{3}^{t}} \\ 0, & \text { if } \times \boldsymbol{F}^{i} \times_{2} \leq \frac{\lambda_{3}}{\mu^{t}}\end{cases}
$$

where $\boldsymbol{F}^{i}$ is the $i$ th row of matrix $\boldsymbol{F}$. Here, we denote the solution of $\boldsymbol{E}$ as $H_{\left(\lambda_{3} / \mu^{t}\right)}(\boldsymbol{F})$ for convenience.

After we optimize variables $\boldsymbol{P}, \boldsymbol{Z}, \boldsymbol{Q}$, and $\boldsymbol{E}$, the ADMM algorithm also needs to update the Lagrange multipliers $\boldsymbol{C}_{1}, \boldsymbol{C}_{2}, \boldsymbol{C}_{3}$, as well as parameter $\mu$, for faster convergence.

The detailed procedures of solying the proposed optimization problem (8) are described in Algorithm 1.

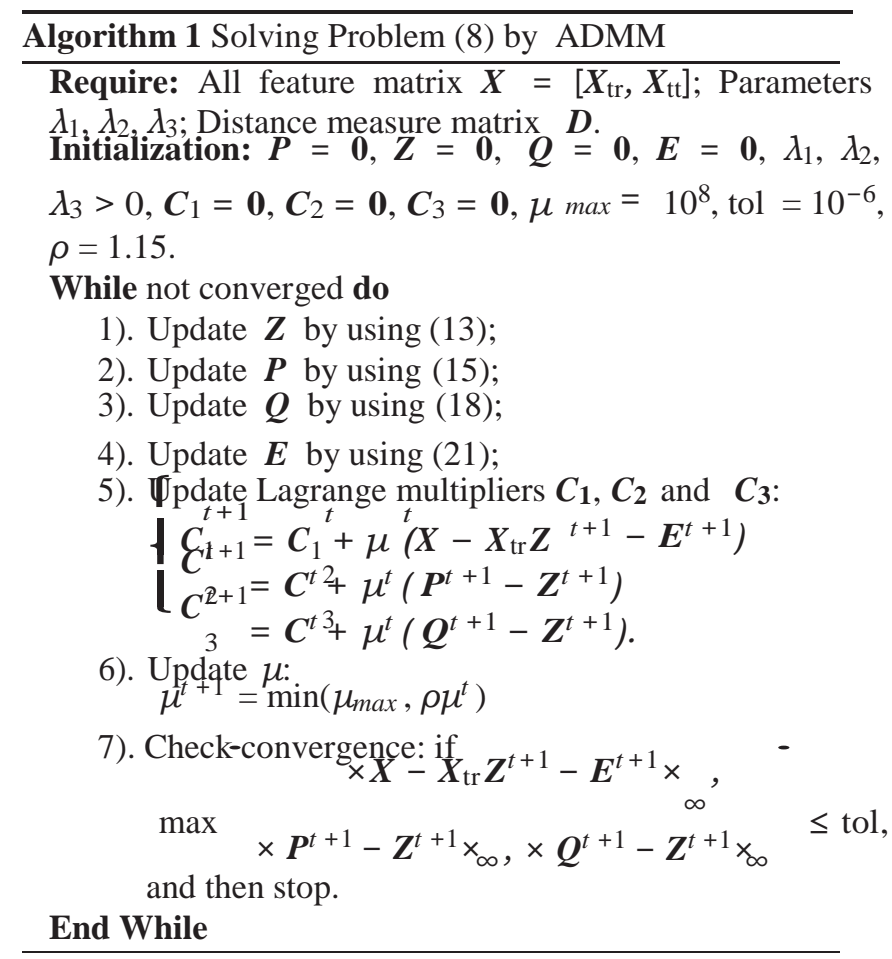

\section{B. Recognition Method}

When problem (8) is optimized by exploiting Algorithm 1, the discriminant data representation $\boldsymbol{Z}=\left[\boldsymbol{Z}_{\mathrm{tr}}, \boldsymbol{Z}_{\mathrm{tt}}\right]$ is obtained. We directly employ a simple linear classifier to perform final recognition [10]. A linear classifier $\boldsymbol{W}$ is learned based on the training data representation $\boldsymbol{Z}_{\mathrm{tr}}$ and its corresponding 
label matrix $L \in \mathrm{K}^{C \times n}$ of training samples. The following optimization problem is considered:

$$
\hat{\boldsymbol{W}}=\underset{\boldsymbol{W}}{\arg \min } \times \boldsymbol{L}-\boldsymbol{W} \boldsymbol{Z}_{\mathrm{tr}} \times_{F}^{2}+\boldsymbol{V} \times \boldsymbol{W} \times{ }_{F}^{2}
$$

where $Y$ is a positive regularization parameter. It is easy to verify that problem (22) has the closed-form solution, that is

$$
\hat{\boldsymbol{W}}=\boldsymbol{L} \boldsymbol{Z}_{\mathrm{tr}}^{T^{-}} \boldsymbol{Z}_{\mathrm{tr}} \boldsymbol{Z}_{\mathrm{tr}}^{T}+\gamma \boldsymbol{I}^{-1} .
$$

The identity of test sample $\boldsymbol{y}$, the $i$ th sample from the test data set, is determined by judging

$$
\operatorname{label}(y)=\arg \max _{j}\left(\hat{W} z^{i}\right)
$$

where $z^{i}$ is the $i$ th column of matrix $\boldsymbol{Z}_{\mathrm{tt}}$. The complete procedures of our BDLRR model for recognition are summarized in Algorithm 2.

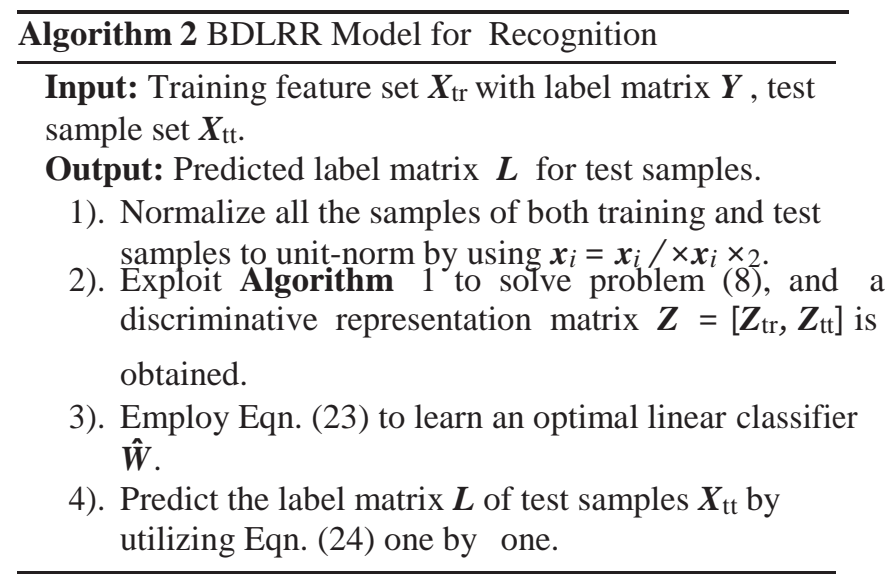

\section{Convergence Analysis}

To solve the proposed formulation (8), an iterative update scheme, the ADMM algorithm, is developed as shown in Section IV-A. This section presents a theoretical convergence proof of the proposed Algorithm 1.

Proposition 1: Algorithm 1 is equivalent to a two-block ADMM

The classical ADMM is intended to solve problems in the form

$$
\min _{z \in \mathrm{R}^{n}, \boldsymbol{w} \in \mathrm{R}} f(z)+h(\boldsymbol{w}) \text { s.t. } \boldsymbol{R} \boldsymbol{z}+\boldsymbol{T} \boldsymbol{w}=\boldsymbol{u}
$$

where $\boldsymbol{R}_{\in \mathrm{K}}{ }^{p \times n}, \boldsymbol{T} \in \mathrm{K}{ }^{p \times m}, \boldsymbol{u} \in \mathrm{K}^{p}$, and $f$ and $h$ are convex functions. It is apparent that ADMM for problem (25) can be directly extended to solve the matrix optimization problem as follows:

$$
\boldsymbol{Z} \in \mathrm{R}_{n \times N} \min _{, \boldsymbol{W} \in \mathrm{R}}^{m \times N} f(\boldsymbol{Z})+h(\boldsymbol{W}) \text { s.t. } \boldsymbol{R} \boldsymbol{Z}+\boldsymbol{T} \boldsymbol{W}=\boldsymbol{U}
$$

where $\boldsymbol{U} \in \mathrm{R}^{p \times N}$. The augmented Lagrangian of problem (26), in the method of multipliers, is formulated as

$$
\begin{aligned}
& L_{\mu}(\boldsymbol{Z}, \boldsymbol{W}, \boldsymbol{C})=f(\boldsymbol{Z})+h(\boldsymbol{W}) \stackrel{\mu}{+} \times \boldsymbol{R} \boldsymbol{Z}+\boldsymbol{T} \boldsymbol{W}-\boldsymbol{U} \times{ }_{F} \\
&+(\boldsymbol{C}, \boldsymbol{R} \boldsymbol{Z}+\boldsymbol{T} \boldsymbol{W}-\boldsymbol{U})(27)
\end{aligned}
$$

where $\boldsymbol{C} \in \mathrm{K}^{p \times N}$ is the Lagrangian multiplier, and $\mu$ is a penalty coefficient.

It should be noted that problem (9) is a special case of problem (26). Specifically, it can be verified that the constraints in (9) can be transformed into the form of $R \boldsymbol{Z}+\boldsymbol{T} \boldsymbol{W}=\boldsymbol{U}$, where $\boldsymbol{R}=\left(\begin{array}{c}-\boldsymbol{I}_{n} \\ \boldsymbol{I}_{\mathrm{tr}}^{n}\end{array}\right), \boldsymbol{T}=\left[\begin{array}{lll}\boldsymbol{I}_{n} & & \\ & \boldsymbol{I}_{n} & \boldsymbol{I}_{d}\end{array}\right], \boldsymbol{W}=\left(\begin{array}{l}\boldsymbol{P} \\ \boldsymbol{Q}\end{array}\right)$, $\boldsymbol{U}=\underset{\boldsymbol{X}}{\left(\begin{array}{l}\mathbf{8} \\ \mathbf{X}\end{array}\right)}$, and $\boldsymbol{I}_{n}$ is an $n \times n$ identity matrix. In this way, problem (9) is reformulated as problem (26). Moreover, ADMM updates two primal variables in an alternating fashion, and iteratively solves problem (27) as follows:

$$
\begin{aligned}
\boldsymbol{Z}^{t+1} & =\arg \min _{\boldsymbol{Z} \in \mathrm{R}^{{ }^{*} \times}} L_{\mu}\left(\boldsymbol{Z}, \boldsymbol{W}^{t}, \boldsymbol{C}^{t}\right) \\
\boldsymbol{W}^{t+1} & =\arg \min _{\boldsymbol{W} \in \mathrm{R}^{m} \times} L_{\mu}\left(\boldsymbol{Z}^{t+1}, \boldsymbol{W}, \boldsymbol{C}^{t}\right) \\
\boldsymbol{C}^{t+1} & =\boldsymbol{C}^{t}+\mu\left(\boldsymbol{R} \boldsymbol{Z}^{t+1}+\boldsymbol{T} \boldsymbol{W}^{t+1}-\boldsymbol{U}\right)
\end{aligned}
$$

which have the same updating procedures as Algorithm 1 in Section IV-A. In fact, we can see that the optimization of $\boldsymbol{Z}$ in (28a) is equivalent to optimize $\boldsymbol{Z}$ in (11). Furthermore, it is very important that when fixing $\boldsymbol{Z}$, solutions of $\boldsymbol{P}$ in (15), $\boldsymbol{Q}$ in (18), and $\boldsymbol{E}$ in (21), are independent on one another, for instance, computation of $\boldsymbol{E}^{t+1}$ only depends on $\boldsymbol{Z}^{k+1}$ and $\boldsymbol{C}^{k+1}$ rather than $\boldsymbol{P}^{k+1}$ or $\boldsymbol{Q}^{k+1}$. Hence, the optimizations of $\boldsymbol{P}, \boldsymbol{Q}$ and $\boldsymbol{E}$ can be accumulated in $\boldsymbol{W}$ by using (28b), updating of which is the same as fashion of Jacobian iterative method. In this way, problem (9) is a special case of classical ADMM problem (26), and the proposed optimization algorithm shown in Algorithm 1 has the same optimization style of classical ADMM (28). Therefore, the proposed optimization algorithm shown in Algorithm 1 is equivalent to a two-block ADMM, the global convergence of which is theoretically guaranteed [29]-[31]. The convergence nature of the proposed optimization algorithm is given by Theorem 1 .

Theorem 1 [30], [31]: Consider the problem (25), where $f(\boldsymbol{Z})$ and $h(\boldsymbol{W})$ are closed proper convex functions, $\boldsymbol{R}$ has full column rank and $h(\boldsymbol{W})+\times \boldsymbol{T} \boldsymbol{W} \times{ }_{F}^{2}$ is strictly convex. Let $\boldsymbol{C}^{0}$ and $\boldsymbol{W}^{0}$ be arbitrary matrix and $\mu>0$. Assume that we have the sequences $\left\{V_{\infty}\right\}$ and $\left\{v_{t}\right\}$ such that $V_{t} \geq 0$ and $v_{t} \geq 0$,

$$
\begin{aligned}
& t=0 V_{t}<\infty \text { and }{ }_{t=0} v_{t}<\infty \text {. Suppose that } \\
& \boldsymbol{Z}^{t+1}-\min _{\boldsymbol{Z}} f(\boldsymbol{Z})+\frac{\mu}{2} \times \boldsymbol{R} \boldsymbol{Z}+\boldsymbol{T} \boldsymbol{W}^{t}-\boldsymbol{U} \times{ }_{F} \\
& +\left(\boldsymbol{C}^{t}, \boldsymbol{R Z}\right)_{F} \leq \gamma_{t} \\
& \boldsymbol{W}^{t+1}-\min _{\boldsymbol{W}} h(\boldsymbol{W})+\frac{\mu}{2} \times \boldsymbol{R} \boldsymbol{Z}^{t+1}+\boldsymbol{T} \boldsymbol{W}-\boldsymbol{U} \times_{\hat{F}} \\
& +\left(\boldsymbol{C}^{t}, \boldsymbol{T W}\right) \leq v_{t} \\
& \boldsymbol{C}^{t+1}=\boldsymbol{C}^{t}+\mu\left(\boldsymbol{R} \boldsymbol{Z}^{t+1}+\boldsymbol{T} \boldsymbol{W}^{t+1}-\boldsymbol{U}\right) .
\end{aligned}
$$

If there exists a saddle point of $L_{\mu}(\boldsymbol{Z}, \boldsymbol{W}, \boldsymbol{C})(27)$, then $\boldsymbol{Z}^{k} \rightarrow$ $\boldsymbol{Z}^{*}, \boldsymbol{W}^{k} \rightarrow \boldsymbol{W}^{*}$, and $\boldsymbol{C}^{k} \rightarrow \boldsymbol{C}^{*}$, where $\left(\boldsymbol{Z}^{*}, \boldsymbol{W}^{*}, \boldsymbol{C}^{*}\right)$ is such a saddle point. On the other hand, if no such saddle point 
exists, then at least one of the sequences $\left\{\gamma_{t}\right\}$ or $\left\{v_{t}\right\}$ must be unbounded.

Clearly, the optimization results shown in Section IV-A indicate that the proposed method exists an optimal solution according to [32, Proposition 1.1.5], and the values sequences $\left\{\gamma_{t}\right\}$ and $\left\{v_{t}\right\}$ are directly set to zeros in Algorithm 1. Therefore, the convergence nature of our optimization method is demonstrated. Moreover, we empirically show in Section V-F that the experimental convergence of the resulting ADMM is well preserved.

\section{Computational Complexity Analysis}

In this section, the computational complexity for Algorithm 1 is presented, and it is easy to see that the recognition process of Algorithm 2 is very efficient, which is linear with the sample number. More specifically, the major computation cost of Algorithm 1 is in steps 1-4, which require computing the SVD and matrix computation operation. Thus, they will be time-consuming when the number of training samples $n$ and the total number of samples $N$ are very large. In particular, computing SVD decomposition of matrix $\boldsymbol{P} \in \mathrm{R}^{n \times N}$ needs the complexity of $O\left(n^{2} N\right)(N>n)$. Note that due to the matrix inverse calculation, calculating $\boldsymbol{Z}$ will scale in about $O\left(n^{2} d+n^{2} N\right)$, where $d$ is the dimensionality of the samples. The computational complexity of step 3 is $O(n N)$, and computing $\boldsymbol{E}$ in step 4 costs $O(d N)$. Therefore, the total computational complexity of BDLRR is $O_{\kappa}\left(2 n^{2} N+n^{2} d+d N+n N\right)$, where $\kappa$ is the number of iterations.

In comparison, the computation burden of the SRC methods, such as SRC, LRSI, and LatLRR, is $O\left(n^{2}(N-n) d\right)$ by solving $(N-n)$ independent $l_{1}$-norm minimization problems in an iterative optimization manner [3], [16], [22], which is slower than that of our method. The computation complexities of regression methods, such as low-rank linear regression (LRLR) and Low-rank robust regression (LRRR), are $O\left(d n+n^{2} d\right)$, which is a little faster than our method. The low-rank and sparse representation-based methods, such as nonnegative low-rank representation sparse (NNLRS), supervised regularization-based robust subspace (SRRS), CBDS, and our BDLRR, need to simultaneously compute SVD of feature matrix and solve a simple soft-thresholding problem, and a linear classification algorithm is used to predict final labels of test data. Generally, the overall computation burden of our BDLRR is the same as those of the low-rank sparse representation learning methods.

\section{E. Out-of-Sample Extension}

It is worth noting that the LRR-based methods have been extensively studied, but how to address the out-of-sample problem, the capability of dealing with new data instances, is much less well-solved. The stage of BDLRR mentioned previously only obtains the discriminative representations of the available samples $\boldsymbol{X} \in \mathrm{R}^{d \times N}$. However, given unseen instances outside the training and test data, it would be unrealistic and time-consuming to reimplement the whole model to produce the representations of novel images. In this section, we will show that the proposed BDLRR method can naturally cope with the out-of-sample examples to learn discriminative visual representations.

Suppose that we have obtained the optimal block-diagonal representation $Z \in \mathrm{R}^{n \times N}$ from the available samples $\boldsymbol{X}$ over $\boldsymbol{X}_{\text {tr }} \in \mathrm{R} \quad$ using the proposed model (8). Now, we extend the proposed BDLRR method to learn preferable representation of a novel image $\boldsymbol{b} \in \mathrm{R}^{d \times 1}$ in the original observed space. Specifically, we aim at learning the discriminative representation $z$ for $\boldsymbol{b}$ over $\boldsymbol{X}_{\text {tr }}$ while fixing the previously learned representation $\boldsymbol{Z}$. Therefore, adding terms for a novel data point $\boldsymbol{b}$ in model (8) and keeping the already learned variables, the objective function of the augmented BDLRR is formulated as

$$
\begin{aligned}
& \left.\left.\min _{z, \boldsymbol{e}} \times[\boldsymbol{Z}, \boldsymbol{z}] \times_{*}+\lambda_{1} \times \hat{\boldsymbol{A}} \text { S( } \boldsymbol{Z}, \boldsymbol{z}\right] \times_{F}+\lambda_{2} \times \hat{\boldsymbol{D}} \text { SQ } \boldsymbol{Z}, \boldsymbol{z}\right] \times_{1} \\
& +\lambda_{3} \times[\boldsymbol{E}, \boldsymbol{e}] \times 21 \\
& \text { s.t. }[\boldsymbol{X}, \boldsymbol{b}]=\boldsymbol{X}_{\mathrm{tr}}[\boldsymbol{Z}, z]+[\boldsymbol{E}, \boldsymbol{e}]
\end{aligned}
$$

where $\hat{\boldsymbol{A}}=\left[\boldsymbol{A}, \mathbf{1}_{n} \mathbf{1}_{N+1-n}^{T}\right], \boldsymbol{A}$ is defined as in (8), $\boldsymbol{D} \in$ $\mathrm{R}^{n \times(N+1)}$ is the distance metric between the training samples $\boldsymbol{X}_{\text {tr }}$ and all samples $[\boldsymbol{X}, \boldsymbol{b}]$, and $\boldsymbol{e}$ is the representation error of $\boldsymbol{b}$ over $\boldsymbol{X}_{\text {tr. }}$. We argue that $\times[\boldsymbol{Z}, \boldsymbol{z}] \times_{*}=\times \mathbf{Z} \times_{*}$. Particularly, for the learned representation $\boldsymbol{Z} \in \mathrm{R} n \times N(n<N)$, it is easy to find that the linear problem for $\boldsymbol{a}$ in $\boldsymbol{z}=\boldsymbol{Z} \boldsymbol{a}$ is an underdetermined system for practical data. Generally speaking, $z=Z a$ has infinitely many solutions in practice [33]. Provided $n \quad N$, the matrix $\boldsymbol{Z}$ is row full rank and $\boldsymbol{z}=\boldsymbol{Z a}$ has solution. In this way, the singular values of matrix $\boldsymbol{Z}$ coincide with those of $[Z, z]$, which means $\operatorname{rank}([Z, z])=\operatorname{rank}(Z)$. Therefore, $\times[\boldsymbol{Z}, z] \times_{*}=\times \boldsymbol{Z} \times_{*}$ and it does not change for practical data in (32). By removing the irrelevant terms with respective to the variables $\boldsymbol{z}$ and $\boldsymbol{e}$, it is easy to check that problem (32) will be degenerated to the following formulation:

$$
\begin{aligned}
& \min _{\boldsymbol{z}, \boldsymbol{e}} \lambda_{1} \times \boldsymbol{z}_{2}+\lambda_{2} \times \boldsymbol{d} S_{\boldsymbol{z}} \times \times_{1}+\lambda_{3} \times \boldsymbol{e} \times 2 \\
& \text { s.t. } \boldsymbol{b}=\boldsymbol{X}_{\mathrm{tr}} \boldsymbol{z}+\boldsymbol{e}
\end{aligned}
$$

which can be equivalently reformulated as

$$
\begin{aligned}
& \min _{z, \boldsymbol{e}} \lambda_{1} \times z \times \times_{2}^{2}+\lambda_{2} \times \boldsymbol{d}\left(S_{z} \times \times_{1}+\lambda_{3} \times \boldsymbol{e} \times{ }_{2}^{2}\right. \\
& \text { s.t. } \boldsymbol{b}=\boldsymbol{X}_{\text {tr }} \boldsymbol{z}+\boldsymbol{e}
\end{aligned}
$$

where $\boldsymbol{d}_{i}$ is the distance between $\boldsymbol{x}_{i}$ and $\boldsymbol{b}$. To make problem (34) more compact, it can be rewritten as

$$
\min _{z} \frac{1}{2} \times \boldsymbol{b}-\boldsymbol{X}_{\mathrm{tr}} z \times{ }_{2}^{2}+\frac{\beta_{1}}{2} \times z \times{ }_{2}^{2}+\beta_{2} \times \boldsymbol{d} \mathrm{S} z \times 1
$$

where $\beta_{1}=\lambda_{1} / \lambda_{3}$ and $\beta_{2}=\lambda_{2} / 2 \lambda_{3}$. Apparently, problem (35) is an elastic-net regularized regression problem. For convenient ${ }_{2}$ interpretation, ${ }_{2}$ we denote $g(z)=(1 / 2) \times \boldsymbol{b}-\boldsymbol{X}_{\text {tr }} z \times \times_{2}+\left(\beta_{1} / 2\right) \times z \times \times_{2}$. With some algebra, problem (35) can be approximately transformed to the following optimization problem:

$$
\begin{aligned}
& z^{k+1} \\
& =\arg \min _{z} \beta_{2} \times \boldsymbol{d}\left(S_{z} \times 1+\left(\nabla_{z} g(z), z-z\right)+{ }_{k} \overline{\times z}-z \times_{2}\right. \\
& =\arg \min _{z} \beta_{2} \times \boldsymbol{d}\left(S z \times x_{1}+\overline{2} \times z-z+\nabla_{z} g(z) / \eta \times \times_{2}+\right.\text { const }
\end{aligned}
$$


where $z^{k}$ is the $k$ th iteration of $\boldsymbol{z}$, and $\eta=\times \boldsymbol{X}_{\text {txx }}^{2}$ is a fixed step size in this paper. Similar to problem (18), the optimal solution of the $i$ th entry of $z$ is calculated by using $z_{i}^{k+1}=$ $S_{\left(\beta_{2} d_{i} / \eta\right)}\left(\left[z^{k}-\nabla_{z} g\left(z^{k}\right) / \eta\right]_{i}\right)$. After obtaining the optimal solution $z$, we identify the new data instance $\boldsymbol{b}$ by employing (24), i.e., label $(\boldsymbol{b})=\arg \max _{j}(\hat{\boldsymbol{W}} z)$. The promising recognition results can be guaranteed based on the observation that the discriminative block-diagonal training representations are learned in the training stage. Therefore, based on the proposed BDLRR model, the problem of recognizing new instances outside the training and test samples is well addressed.

\section{F. Discussion}

As we know, BDLRR simultaneously takes advantages of supervised information, i.e., label information, and semisupervised learning superiority, i.e., learning training and test representations in one formulation. Moreover, our method intrinsically inherits the superiorities of sparse, low-rank, structured, and elastic-net representation learning techniques. This characteristic naturally differentiates it from previous works, yielding superior recognition results. In this section, we establish the relationships between the proposed BDLRR method and some related discriminative LRR methods, such as the NNLRS method [25], the structured sparse and lowrank representation (SSLR) method [10], and the very recently proposed SRRS method [24].

1) Connection to the NNLRS Method: The NNLRS method focuses on constructing the informative graph by jointly considering the low-rank and sparse representation to capture the global and local structures of data, respectively. Specifically, the objective function of NNLRS is formulated as

$$
\begin{aligned}
& \min _{\boldsymbol{Z}, \boldsymbol{E}} \times \boldsymbol{Z} \times \times_{*}+\lambda_{2} \times \boldsymbol{Z} \times{ }_{1}+\lambda_{3} \times \boldsymbol{E} \times 21 \\
& \text { s.t. } \boldsymbol{X} \mathbf{t r}=\boldsymbol{X}_{\mathbf{t r}} \boldsymbol{Z}+\boldsymbol{E}, \boldsymbol{Z} \geq \mathbf{0} .
\end{aligned}
$$

The rationale of NNLRS is under the guidance of the observation that the sparse constraint ensures each sample connected to only few other samples resulting in sparse representation, while the low-rank constraint enforces the learned representation from the same class with high correlations. In other words, NNLRS is designed to capture the global structure of the training data using the low-rank property, and the locality information of each data vector is interpolated into NNLRS by introducing the sparse term. Proposition 2shows the close relationship between the proposed BDLRR method and the LRR and NNLRS methods.

Proposition 2: The proposed BDLRR method is a generalized but discriminative LRR learning model, and both of LRR and NNLRS are the special cases of the proposed BDLRR method.

Proof: The detailed proof of Proposition 2 is moved to Supplementary file due to page limitation of this paper.

2) Comparison With the SSLR Method: SSLR first learns a structured low-rank sparse dictionary by imposing an ideal representation regularization term, and then, an SLRR is achieved based on the learned dictionary. The objective function of
SSLR is

$$
\begin{aligned}
& \min _{\boldsymbol{Z}, \boldsymbol{E},} \times \boldsymbol{Z} \times \times_{*}+\lambda_{1} \times \boldsymbol{Z} \times_{1}+\lambda_{2} \times \boldsymbol{E} \times{ }_{1}+\lambda_{3} \times \boldsymbol{Z}-\boldsymbol{Z} \times_{F} \\
& \text { s.t. } \boldsymbol{X}_{\mathrm{tr}}=\boldsymbol{Z}+\boldsymbol{E}
\end{aligned}
$$

where is the learned dictionary. $\boldsymbol{Q}$ is the ideal data representation of training samples, i.e., $\left.\begin{array}{ccc}\mathbf{1}_{s_{1}} \mathbf{1}_{s 1}^{T} & \ldots & 0 \\ \ddots & \ddots & \vdots \\ 0 & \ldots & \mathbf{1}_{S C} \mathbf{1}_{S C}^{T}\end{array}\right]$, where $s_{i}$ is the number of the $i$ th class of . By solving the optimization problem (38), the learned dictionary is obtained, and then, representations of the training and test data are, respectively, achieved by directly removing the ideal representation term from (38), resulting in the following optimization problem:

$$
\min _{\boldsymbol{Z}, \boldsymbol{E}} \times \boldsymbol{Z} \times{ }_{*}+\lambda_{1} \times \boldsymbol{Z} \times{ }_{1}+\lambda_{2} \times \boldsymbol{E} \times 1 \text { s.t. } \boldsymbol{B}=\boldsymbol{Z}+\boldsymbol{E}
$$

where $\boldsymbol{B}$ is the observations, i.e., $\boldsymbol{X}_{\mathrm{tr}}$ or $\boldsymbol{X}_{\mathrm{tt}}$. Although the experimental results reported in [10] are good, we hold the view that enforcing the representation approximal to the ideal representation matrix $\boldsymbol{Q}$ is questionable, because it is impossible to regularize all the training samples of the same class to have the same representation codes. Moreover, the solution of learning by solving problem (38) sensitively depends on the initialization because of the nonconvex optimization. Furthermore, learning representations of the training and test data are divided into two separate stages, and there are, respectively, three and two parameters in (38) and (39), which are very difficult to tune.

In contrast, our method is reasonable and discriminative. BDLRR first shrinks the off-block-diagonal elements to eliminate the unfavorable representations resulting in marginalized interclass representations and highlights the block-diagonal elements yielding compact intraclass representations. In this way, the discriminative constraints in BDLRR simultaneously separate the common visual representations from different classes, and effectively prevent zero entities from appearing in the class-specific representations. Moreover, we believe that it is significant for recognition that the learned representations of training and testing samples should be consistent. To this end, BDLRR builds the representation bridge between the training and test samples by imposing the low-rank and locality coherence property. Thus, the proposed BDLRR method unifies the discriminative representations of training and test data into one robust learning framework such that better recognition results are achieved.

3) Comparison With the SRRS Method: The main objective of SRRS is dedicated to learning a discriminative subspace from the clean data recovered by using the LRR constraint. The main idea of SRRS is to remove noise from contaminated data depending on the denoising capability of the LRR, and then, the discriminative subspace is learned based on the recovered clean data. The objective function of SRRS is

$$
\begin{aligned}
& \min _{\boldsymbol{Z}, \boldsymbol{E}, \boldsymbol{P}} \mid \boldsymbol{Z} \times_{*}+\lambda_{2} \times \boldsymbol{E} \times_{21}+\eta \times \boldsymbol{P}^{T} \boldsymbol{X} \boldsymbol{Z}_{\times_{F}^{2}} \\
& \quad+\lambda_{1}\left[\operatorname{tr}\left(S_{b}\left(\boldsymbol{P}^{T} \boldsymbol{X} \boldsymbol{Z}\right)\right)-\operatorname{tr}\left(S_{w}\left(\boldsymbol{P}^{T} \boldsymbol{X} \boldsymbol{Z}\right)\right)\right] \\
& \text { s.t. } \boldsymbol{X}_{\mathrm{tr}}=\boldsymbol{X}_{\mathrm{tr}} \boldsymbol{Z}+\boldsymbol{E}, \boldsymbol{P}^{T} \boldsymbol{P}=\boldsymbol{I}
\end{aligned}
$$


where $S_{b}(\cdot)$ and $S_{w}(\cdot)$ are, respectively, the between-class and within-class scatter matrices, and $\eta$ is a balance parameter.

Apparently, our method is different from SRRS. First, SRRS directly utilizes the clean data $\boldsymbol{X Z}$ to perform discriminant analysis, which means that the performance of SRRS is greatly subject to the denoising ability of LRR. However, BDLRR aims at directly learning discriminative representations from data by imposing the discriminant constraints, which are not confined to any other conditions. Moreover, SRRS is a subspace learning method, and then, the dimension selection of the final representations is very important for recognition. However, BDLRR directly learns discriminative representations from data, and recognition is performed on the optimal representations without bearing the burden of dimension selection. In addition, our BDLRR method jointly learns the representations of training and test data, whereas the test representations of SRRS is achieved by using $\boldsymbol{P} \boldsymbol{X}_{\mathrm{tt}}$, which cannot capture the component connections between the learned representations of the training and test data. Therefore, the proposed BDLRR method is more robust and discriminative than SRRS, which is also verified by the subsequent experimental results.

\section{EXPERIMENTAL VALIDATION}

In this section, the performance of the proposed BDLRR method is evaluated for different recognition tasks. Extensive experiments are performed on different types of data sets to demonstrate the effectiveness and superiority of the proposed method in comparison with the state-of-the-art recognition methods. Subsequently, the algorithmic convergence and the selection of parameters are well analyzed.

\section{A. Experimental Setup}

We test our method on eight benchmark data sets for three basic recognition tasks. Moreover, we compare with some state-of-the-art recognition methods, including representationbased methods (such as SRC [3], LLC [4], CRC [16], and LRC [18]), low-rank criterion-based methods (such as LatLRR [7], LRLR [20], LRRR [20], RPCA [21], LRSI [22], CBDS [23], SRRS [24], and NNLRS [25]), and conventional classification methods, such as support vector machine (SVM) [34] with Gaussian kernel. We randomly select several images per class to construct the training data set, and the rest of images are regarded as the test set. All the selection processes are repeated ten times, and the average recognition accuracies are reported for all the methods.

For fair comparison in all experiments, we use the MATLAB codes from the corresponding authors with the default or optimal parameter settings, or directly cite the experimental results from their original papers. More specifically, for RPCA [21], we first use the original RPCA algorithm on both training and test data sets to eliminate some noise and corrupted terms, and, then, exploit SRC [3] for recognition. For LatLRR [7], the learned salient features are used for recognition. For SVM, the LibSVM software [34] is used for multiclass recognition, where the important regularization parameter $C$ in SVM is selected by cross validation from the candidate set $\{0.01,0.1,1.0,10.0,100.0,1000.0\}$. The parameters of our method, i.e., $\lambda_{1}, \lambda_{2}$, and $\lambda_{3}$, are tuned to achieve the best performance via fivefold cross validations from $[0.1,0.5,1,5,10,15,20,25]$. To guarantee the same experimental settings between all the compared methods and our method on each benchmark, we reimplemented all the algorithms using respective optimal parameters via the crossvalidation strategy, and the training and test samples were randomly selected from each data set ten times. Since the scene character recognition data sets have the standard splits of the training and test data, we directly employ the full training and test data for recognition, and the compared experimental results are cited from the original papers. Similarly, for scene recognition, experiments are performed with the same experiment protocols as that of the LC-KSVD method [39], and we directly cite some experimental results from the original papers. For the compared methods that are not included in [39], we rerun them following the same experimental settings. Therefore, all the methods presented in this paper are performed on the same test bed for each data set such that our experimental results are convincing and reliable. All algorithms are implemented with MATLAB 2013a, and the $M A T L A B$ code of the proposed method has been released at http://www.yongxu.org/lunwen.html.

\section{B. Experiments for Face Recognition}

In this section, we perform experiments on four face image data sets, including the extended YaleB [35], CMU PIE [36], AR [37], and Labeled Faces in the Wild (LFW) [38] data sets.

1) Extended YaleB Database: The extended YaleB database is composed of 2414 face images of 38 subjects, where each person has 59-64 near frontal images under different illumination conditions. All the images for our experiments on this database have been resized to $32 \times 32$ pixels. For all the compared methods, the suggested parameters from the corresponding papers are used for recognition. For the LLC [4] method, we directly treat the training samples as the bases, and the coding coefficients are obtained using the approximated LLC strategy. The number of neighbors of LLC is set to 15 for this data set, which can achieve the highest recognition accuracies. In the experiments, we randomly select $20,25,30$, and 35 images per subject for training and the rest for testing. The recognition accuracies of different methods on this database are shown in Table I. Note that the mean classification accuracies and the corresponding standard deviations (acc \pm std) are reported, and the bold numbers suggest the highest recognition accuracies. From Table I, it is easy to find that our method can consistently achieve the highest recognition results, and outperforms the other 11 competing methods significantly, even when using a small number of training samples. Moreover, the experimental results also validate that our method has an outstanding capability on overcoming the challenges of illumination and expression variations.

2) $C M U$ PIE Database: The CMU PIE face database contains more than 40000 face images of 68 individuals in total. In our experiments, we utilize the images under five near frontal poses ( 05 , C07, C09, C27, and C29), and then 
TABLE I

ReCognition ACCuracies (MEAN \pm StD \%) OF DifFERENT Methods With DifFERENT NuMBERS OF TRAINING SAmples on the EXTENDED YaleB Database

\begin{tabular}{|c|c|c|c|c|}
\hline Alg. & 20 & 25 & 30 & 35 \\
\hline \hline LRC & $92.15 \pm 0.95$ & $93.55 \pm 0.65$ & $94.55 \pm 0.68$ & $95.49 \pm 0.55$ \\
CRC & $94.36 \pm 1.17$ & $95.89 \pm 0.91$ & $97.14 \pm 0.75$ & $97.93 \pm 0.55$ \\
SRC & $93.73 \pm 0.70$ & $95.58 \pm 0.26$ & $96.37 \pm 0.45$ & $97.13 \pm 0.42$ \\
LLC & $91.60 \pm 0.50$ & $94.20 \pm 0.49$ & $95.29 \pm 0.38$ & $96.05 \pm 0.51$ \\
SVM & $92.81 \pm 0.68$ & $95.20 \pm 0.44$ & $96.11 \pm 0.41$ & $96.70 \pm 0.69$ \\
RPCA & $93.58 \pm 0.61$ & $95.51 \pm 0.36$ & $96.70 \pm 0.46$ & $96.96 \pm 0.49$ \\
LatLRR & $93.05 \pm 0.95$ & $93.91 \pm 0.68$ & $95.03 \pm 0.83$ & $97.14 \pm 0.36$ \\
LRLR & $83.91 \pm 1.53$ & $85.15 \pm 1.50$ & $85.49 \pm 1.05$ & $85.95 \pm 1.47$ \\
LRRR & $83.95 \pm 0.82$ & $85.66 \pm 0.93$ & $86.21 \pm 0.99$ & $86.55 \pm 0.81$ \\
CBDS & $95.99 \pm 1.11$ & $96.56 \pm 0.85$ & $97.61 \pm 0.82$ & $98.13 \pm 0.55$ \\
LRSI & $94.19 \pm 0.44$ & $96.28 \pm 0.61$ & $96.99 \pm 0.57$ & $97.72 \pm 0.48$ \\
NNLRS & $94.35 \pm 0.79$ & $96.06 \pm 0.63$ & $97.02 \pm 0.61$ & $97.62 \pm 0.42$ \\
SRRS & $93.74 \pm 0.86$ & $96.05 \pm 0.95$ & $96.89 \pm 0.84$ & $97.15 \pm 0.58$ \\
\hline BDLRR & $\mathbf{9 6 . 8 9} \pm \mathbf{0 . 6 7}$ & $\mathbf{9 7 . 9 6} \pm \mathbf{0 . 4 2}$ & $\mathbf{9 8 . 7 0 \pm 0 . 4 6}$ & $\mathbf{9 9 . 4 6} \pm \mathbf{0 . 2 9}$ \\
\hline
\end{tabular}

TABLE II

Recognition Accuracies (Mean \pm StD \%) of Different Methods With DifFERENT NuMBERS OF TRAINING SAMPLES ON THE CMU PIE DATABASE

\begin{tabular}{|c|c|c|c|c|}
\hline Alg. & 20 & 25 & 30 & 35 \\
\hline \hline LRC & $90.07 \pm 0.52$ & $92.65 \pm 0.38$ & $94.11 \pm 0.26$ & $94.88 \pm 0.17$ \\
CRC & $92.52 \pm 0.33$ & $93.84 \pm 0.39$ & $94.31 \pm 0.16$ & $95.52 \pm 0.16$ \\
SRC & $92.14 \pm 0.29$ & $93.65 \pm 0.38$ & $94.51 \pm 0.28$ & $95.86 \pm 0.24$ \\
LLC & $91.90 \pm 0.25$ & $93.27 \pm 0.56$ & $94.66 \pm 0.41$ & $95.26 \pm 0.49$ \\
SVM & $90.69 \pm 0.73$ & $92.78 \pm 0.68$ & $93.19 \pm 0.51$ & $94.10 \pm 0.30$ \\
RPCA & $88.34 \pm 0.32$ & $91.56 \pm 0.18$ & $92.96 \pm 0.22$ & $93.81 \pm 0.36$ \\
LatLRR & $88.84 \pm 0.32$ & $91.96 \pm 0.82$ & $93.26 \pm 0.22$ & $94.41 \pm 0.38$ \\
LRLR & $85.83 \pm 0.56$ & $86.90 \pm 0.45$ & $87.82 \pm 0.49$ & $88.23 \pm 0.42$ \\
LRRR & $85.98 \pm 0.61$ & $86.89 \pm 0.58$ & $88.50 \pm 0.87$ & $89.06 \pm 0.42$ \\
CBDS & $91.81 \pm 0.62$ & $93.50 \pm 0.73$ & $94.45 \pm 0.77$ & $94.90 \pm 0.68$ \\
LRSI & $90.68 \pm 0.56$ & $93.55 \pm 0.69$ & $94.62 \pm 0.54$ & $95.12 \pm 0.26$ \\
NNLRS & $91.72 \pm 0.43$ & $92.04 \pm 0.53$ & $93.55 \pm 0.40$ & $94.38 \pm 0.39$ \\
SRRS & $90.87 \pm 0.61$ & $93.16 \pm 0.45$ & $94.41 \pm 0.35$ & $95.15 \pm 0.27$ \\
\hline BDLRR & $\mathbf{9 4 . 6 7 \pm 0 . 3 1}$ & $\mathbf{9 5 . 7 9} \pm \mathbf{0 . 2 9}$ & $\mathbf{9 6 . 4 6} \pm \mathbf{0 . 1 5}$ & $\mathbf{9 6 . 8 1} \pm \mathbf{0 . 1 4}$ \\
\hline
\end{tabular}

about 170 image samples are obtained for each individual. We randomly select 20, 25, 30, and 35 images from each subject as training samples and the remaining images are regarded as test samples. Each image is cropped and resized to be only $32 \times 32$ pixels. The detailed comparison results obtained using different methods are summarized in Table II. We can see that, with different numbers of training samples per class, our results are always better than those of all the other state-of-the-art methods, which demonstrates the effectiveness of our method.

3) AR Database: The AR face database contains about 4000 color face images of 126 subjects. For each subject, there are 26 images taken in two separate sessions under different conditions. In our experiments, we randomly choose a subset, including 2600 images of 50 female and 50 male subjects. Random face images of the AR face database ${ }^{1}$ are employed in our experiments. Following the implementation in [39], each image is projected onto a 540-D feature vector with a randomly generated matrix with a zero-mean normal distribution. We randomly select 11, 14, 17, and 20 images of each subject as training samples and treat the remaining images as test samples. The experimental results obtained

\footnotetext{
${ }^{1}$ This data set is publicly available from http://www.umiacs.umd.edu/ $\sim$ zhuolin/projectlcksvd.html.
}

TABLE III

RECOGNITION ACCURACIES (MEAN \pm STD \%) OF DiFFERENT METHODS With DifFERENT NuMBERS OF TRAINING SAMPLES ON THE AR DATABASE

\begin{tabular}{|c|c|c|c|c|}
\hline Alg. & 11 & 14 & 17 & 20 \\
\hline \hline LRC & $76.91 \pm 1.33$ & $85.51 \pm 1.20$ & $90.99 \pm 0.97$ & $94.22 \pm 0.76$ \\
CRC & $91.76 \pm 0.77$ & $94.36 \pm 0.97$ & $95.84 \pm 0.76$ & $96.63 \pm 0.87$ \\
SRC & $89.62 \pm 0.74$ & $92.35 \pm 1.29$ & $95.24 \pm 0.67$ & $96.19 \pm 0.75$ \\
LLC & $60.89 \pm 0.97$ & $66.98 \pm 1.13$ & $71.58 \pm 1.32$ & $73.53 \pm 2.15$ \\
SVM & $86.30 \pm 1.33$ & $92.03 \pm 0.77$ & $95.19 \pm 0.88$ & $96.43 \pm 1.26$ \\
RPCA & $84.53 \pm 1.43$ & $88.92 \pm 0.95$ & $92.62 \pm 0.77$ & $94.90 \pm 0.78$ \\
LatLRR & $92.83 \pm 1.06$ & $95.96 \pm 0.70$ & $97.13 \pm 0.85$ & $97.78 \pm 0.56$ \\
LRLR & $88.93 \pm 0.86$ & $93.33 \pm 0.73$ & $94.92 \pm 0.68$ & $96.37 \pm 0.88$ \\
LRRR & $93.82 \pm 0.70$ & $95.42 \pm 0.48$ & $96.47 \pm 0.70$ & $96.88 \pm 0.61$ \\
CBDS & $92.99 \pm 0.59$ & $95.57 \pm 0.60$ & $96.83 \pm 0.63$ & $97.49 \pm 0.82$ \\
LRSI & $86.93 \pm 1.00$ & $90.02 \pm 0.76$ & $93.27 \pm 0.97$ & $94.82 \pm 0.99$ \\
NNLRS & $92.11 \pm 0.70$ & $95.24 \pm 0.49$ & $96.69 \pm 0.56$ & $97.40 \pm 0.65$ \\
SRRS & $87.53 \pm 1.00$ & $93.33 \pm 1.04$ & $96.22 \pm 1.03$ & $97.17 \pm 0.54$ \\
\hline BDLRR & $\mathbf{9 6 . 6 9} \pm \mathbf{0 . 4 1}$ & $\mathbf{9 7 . 9 2 \pm \mathbf { 0 . 3 0 }}$ & $\mathbf{9 8 . 7 2 \pm \mathbf { 0 . 4 2 }}$ & $\mathbf{9 9 . 0 3} \pm \mathbf{0 . 3 8}$ \\
\hline
\end{tabular}

\section{TABLE IV}

RECOGNITION ACCURACIES (MEAN \pm STD \%) OF DiFFERENT Methods With DifFERENT Numbers OF TRAINING SAMPLES ON THE LFW DATABASE

\begin{tabular}{|c|c|c|c|c|}
\hline Alg. & 5 & 6 & 7 & 8 \\
\hline \hline LRC & $29.48 \pm 1.48$ & $33.63 \pm 1.76$ & $35.57 \pm 1.89$ & $37.63 \pm 1.99$ \\
CRC & $29.64 \pm 1.22$ & $31.79 \pm 1.52$ & $32.96 \pm 1.32$ & $33.86 \pm 1.55$ \\
SRC & $29.13 \pm 1.27$ & $32.25 \pm 1.55$ & $33.46 \pm 2.10$ & $36.51 \pm 2.24$ \\
LLC & $27.63 \pm 1.62$ & $29.58 \pm 1.39$ & $31.16 \pm 1.28$ & $31.94 \pm 0.88$ \\
SVM & $30.72 \pm 1.57$ & $33.36 \pm 1.70$ & $36.46 \pm 1.42$ & $37.73 \pm 1.45$ \\
RPCA & $31.55 \pm 1.27$ & $34.17 \pm 1.65$ & $36.68 \pm 1.88$ & $37.99 \pm 1.36$ \\
LatLRR & $30.00 \pm 1.11$ & $33.09 \pm 1.95$ & $35.33 \pm 1.91$ & $37.28 \pm 1.68$ \\
LRLR & $29.68 \pm 1.05$ & $30.18 \pm 1.01$ & $34.55 \pm 1.82$ & $35.39 \pm 2.17$ \\
LRRR & $30.98 \pm 1.28$ & $32.93 \pm 1.70$ & $34.86 \pm 1.03$ & $36.59 \pm 1.87$ \\
CBDS & $34.77 \pm 1.46$ & $36.54 \pm 1.81$ & $37.50 \pm 1.56$ & $38.53 \pm 1.79$ \\
LRSI & $31.57 \pm 2.10$ & $34.42 \pm 1.25$ & $37.18 \pm 0.92$ & $39.25 \pm 1.58$ \\
NNSLR & $34.59 \pm 0.92$ & $35.51 \pm 1.49$ & $36.83 \pm 0.93$ & $39.96 \pm 1.53$ \\
SRRS & $31.67 \pm 1.54$ & $34.29 \pm 1.74$ & $38.06 \pm 1.59$ & $39.43 \pm 1.65$ \\
\hline BDLRR & $\mathbf{3 7 . 8 3} \pm 1.00$ & $\mathbf{4 0 . 9 4 \pm 1 . 7 8}$ & $\mathbf{4 3 . 1 1 \pm 1 . 4 5}$ & $\mathbf{4 4 . 5 1 \pm 1 . 1 5}$ \\
\hline
\end{tabular}

using different recognition methods are shown in Table III. From the results shown in Table III, we know that our method still achieves the best recognition results, which also verifies the fact that the proposed method has particular potential for image recognition. It is notable that even when using smaller number of training samples, the performance gain of our method is still obvious in comparison with other methods.

4) LFW Database: The LFW face database is designed for the study of unconstrained identity verification and face recognition. It contains more than 13000 face images from 1680 subjects pictured under the unconstrained conditions. In our experiments, we employ a subset including 1251 images from 86 people, and each subject has only 10-20 images [40] with an imbalanced number of samples. Each image was manually cropped and resized to $32 \times 32$ pixels. In our experiments, we randomly select 5, 6, 7, and 8 images of each subject as training samples and the remaining face images are treated as test samples. The experimental results of different recognition methods on this data set are presented in Table IV. We can see that the best recognition results are still achieved by our BDLRR method. Especially, the performance of our method has exceedingly advantages for this data set in comparison with the rest of methods. 
TABLE V

RECOGNITION ACCURACIES (MEAN \pm STD \%) OF DifFERENT Methods With DiffERENT NuMBERs OF TRAINING SAMPLES ON THE USPS DATABASE

\begin{tabular}{|c|c|c|c|c|}
\hline Alg. & 30 & 60 & 90 & 120 \\
\hline \hline LRC & $89.53 \pm 0.40$ & $92.68 \pm 0.29$ & $94.17 \pm 0.22$ & $94.94 \pm 0.13$ \\
CRC & $89.53 \pm 0.63$ & $90.79 \pm 0.30$ & $91.47 \pm 0.32$ & $91.71 \pm 0.23$ \\
SRC & $90.06 \pm 0.61$ & $93.46 \pm 0.22$ & $94.87 \pm 0.25$ & $95.38 \pm 0.28$ \\
LLC & $91.30 \pm 0.46$ & $93.72 \pm 0.23$ & $94.78 \pm 0.22$ & $95.42 \pm 0.28$ \\
SVM & $90.77 \pm 0.70$ & $92.67 \pm 0.33$ & $93.59 \pm 0.25$ & $94.01 \pm 0.24$ \\
RPCA & $90.07 \pm 0.29$ & $93.54 \pm 0.34$ & $94.72 \pm 0.12$ & $95.38 \pm 0.20$ \\
LatLRR & $88.75 \pm 0.70$ & $90.26 \pm 0.55$ & $91.08 \pm 0.34$ & $91.56 \pm 0.33$ \\
LRLR & $84.71 \pm 2.02$ & $87.91 \pm 0.82$ & $88.17 \pm 0.76$ & $88.66 \pm 0.47$ \\
LRRR & $86.02 \pm 2.16$ & $88.22 \pm 0.84$ & $88.38 \pm 0.69$ & $88.77 \pm 0.45$ \\
CBDS & $87.80 \pm 0.69$ & $89.46 \pm 0.52$ & $90.46 \pm 0.24$ & $91.54 \pm 0.19$ \\
LRSI & $90.62 \pm 0.41$ & $93.51 \pm 0.31$ & $94.54 \pm 0.17$ & $95.39 \pm 0.18$ \\
NNSLR & $90.54 \pm 0.57$ & $93.00 \pm 0.35$ & $94.03 \pm 0.22$ & $94.88 \pm 0.33$ \\
SRRS & $91.13 \pm 0.20$ & $92.93 \pm 0.36$ & $93.94 \pm 0.21$ & $94.44 \pm 0.20$ \\
\hline BDLRR & $\mathbf{9 2 . 9 0} \pm \mathbf{0 . 3 2}$ & $\mathbf{9 5 . 0 8} \pm \mathbf{0 . 2 7}$ & $\mathbf{9 5 . 9 1} \pm \mathbf{0 . 2 5}$ & $\mathbf{9 6 . 4 1} \pm \mathbf{0 . 2 5}$ \\
\hline
\end{tabular}

\section{Experiments for Character Recognition}

In this section, we evaluate the performance of our method for character recognition. More specifically, three character image data sets are employed for our experiments, including one handwriting data set (i.e., the USPS [41] data set) and two scene character recognition data sets (i.e., the Char74K [42] and SVT [43] data sets). It is worth noting that this paper for the first time learns discriminative data representations for scene character recognition.

1) Handwriting Image Recognition: The USPS Database ${ }^{2}$ refers to numeric data images cropped from the scanning of handwritten digits from envelopes. It consists of 9298 handwritten digits ("0"-"9"). All the images are resized into $16 \times 16$ pixels with 8-b grayscale images. Each digit has about 1100 images. In the experiments, we randomly choose 30, 60, 90, and 120 images of each digit as training samples, and regard the rest of images as test samples. The experimental results of different methods with varying numbers of training samples are shown in Table V. The proposed method performs consistently better than all the compared methods, which further confirms that the proposed method has apparent advantages on recognizing handwriting digit images.

2) Scene Character Image Recognition: Two scene character image data sets are utilized for measuring the effectiveness of our method. As we know, natural scene character recognition is a typical yet challenging pattern recognition task due to the cluttered background, which is very difficult to separate from text. We evaluate the performance of our method in comparison with the state-of-the-art methods experimented on both data sets, including GB [42] (GB+SVM and GB+NN), HOG+NN [43], CoHOG [45], ConvCoHOG [46], PHOG [47], MLFP [48], RTPD [49], GHOG [50], LHOG [50], and SBSTR [51]. All the images in the experiments are first resized into $32 \times 32$ pixels, and gray scale images are used in all the experiments. To make fair comparisons, we directly employ the standard partitions of training and test samples

for each data set as in [44]-[46], and the state-of-the-art algorithms evaluated on respective data sets are directly cited

\footnotetext{
${ }^{2}$ In this paper, the publicly available set is from http://cs.nyu.edu/ $\sim$ roweis/data.html is used.
}

TABLE VI

RECOGNITION ACCURACIES (\%) OF DiffERENT METHODS ON THE SCEne Character Database

\begin{tabular}{|c|c|c|}
\hline \multirow{2}{*}{ Alg. } & \multicolumn{2}{|c|}{ Testing datasets Accuracy } \\
\cline { 2 - 3 } & Char74K-15 & SVT \\
\hline \hline BDLRR & 70 & 79 \\
RPCA+HOG+SRC [44] & 67 & 75 \\
RPCA+HOG+Linear SVM [44] & 63 & 73 \\
RPCA+HOG+SVM(RBF) [44] & 63 & 74 \\
ConvHOG [46] & - & 75 \\
CoHOG [45] & - & 73 \\
PHOG (Chi-Square Kernel) [47] & - & 75 \\
MLFP [48] & 64 & - \\
RTPD [49] & - & 67 \\
GHOG+SVM [50] & 58 & - \\
LHOG+SVM [50] & 60 & - \\
SBSTR [51] & 58 & 68 \\
HOG+NN [43] & 53 & - \\
GB+SVM [42] & 47 & - \\
GB+NN [42] & & \\
\hline
\end{tabular}

from their original papers. For the features ${ }^{3}$ used in our experiments, we exploit the method in a recent paper [44] for feature extraction. Specifically, we first use RPCA [21] to jointly remove noisy pixels and recover clean character images from the blurred or corrupted images, and then, the wellknown HOG method is applied to extract gradient features from the recovered images. The obtained HOG features are utilized for recognition.

a) Char74K database: was collected for the study of recognizing characters in images of natural scenes. An annotated database of images, including English and Kannada characters, was obtained from images captured in Bangalore and India. We mainly focus on the recognition of English characters and digits (i.e., " 0 "-"9," "A"-"Z," and "a"-“ $Z$ ") with 62 classes in total. In our experiments, a small subset is used in our experiments, i.e., Char74K-15, which contains 15 training samples and 15 test samples per class. Table VI presents the recognition results of our method and several recently proposed character recognition methods. From Table VI, we can see that our method can continually achieve the highest recognition results in comparison with the state-ofthe-art methods. Specifically, it is easy to see that our method outperforms the second best algorithm by a large margin of $3 \%$.

b) Street view text (SVT) database: was collected from Google Street View of road-side scenes. All the images are very difficult and challenging to recognize due to the large variations in illumination, character image size, and font size and style. The SVT character data set, which was annotated

in [43], is utilized for evaluating different scene character recognition methods. About 3796 character samples from 52 categories (no digit images) are annotated for recognition.

Moreover, the SVT character data set is more difficult to recognize than the Char $74 \mathrm{~K}$ data set. The experimental results of using different methods on the SVT data set are summa-

rized in Table VI. For this data set, the proposed method significantly outperforms all the other state-of-the-art methods.

\footnotetext{
${ }^{3}$ The features of both data sets are publicly available at http:// www.yongxu.org/databases.html.
} 
TABLE VII

Recognition Accuracies (Mean \pm Std \%) of Different Methods on THE 15 SCEne CATEGORIES DATABASE

\begin{tabular}{|c|c|c|c|}
\hline Alg. & Accuracy & Alg. & Accuracy \\
\hline LLC & 79.4 & SVIV & $\overline{95.0}$ \\
\hline $\mathrm{LLC}^{*}$ & 89.2 & LRSI & 92.4 \\
\hline LRC & 91.9 & CBDS & 95.7 \\
\hline CBC & 92.3 & LRRC [10] & 90.1 \\
\hline SkE & 918 & SLRRR [10] & $91: 3$ \\
\hline LRLR & 94.4 & SRRS & 95.9 \\
\hline LRRR & 87.2 & Lazebnik [52] & 81.4 \\
\hline RPCA & 92.1 & $\operatorname{Lian}[53]^{-1}$ & $\begin{array}{l}81.4 \\
86.4\end{array}$ \\
\hline NNLRS & 86.4 & Yang [54] & 88.3 \\
\hline LatLRR & 91.5 & Boureau [55] & $84: 3$ \\
\hline LC_KSVD1 [39] & 90.4 & Gao [56] & 89.7 \\
\hline LC_KSVD2 [39] & 92.9 & BDLRR & 98.9 主 8.19 \\
\hline
\end{tabular}

We can see that the proposed method (BDLRR) achieves $79 \%$ accuracy, which improves the accuracy by $4 \%$ in comparison with the second best competitors such as SRC used in [44], CoHOG [45], and PHOG [47].

\section{Experiments for Scene Recognition}

The performance of the proposed method for scene recognition is evaluated on the 15 scene categories database [52]. It contains 4485 scene images falling into 15 categories, including livingroom, bedroom, mountain, outdoor street, suburb, industrial, kitchen, opencountry, coast, forest, highway, insidecity, tallbuilding, office, and store. The features ${ }^{4}$ of 15 scene categories provided in [39] is employed for recognition. More specifically, the obtained features are processed as the following steps. First, the spatial pyramid feature with a four-leel spatial pyramid [52] is computed on an SIFTdescriptor codebook with a size of 200 , and then, the spatial pyramid features are reduced to 3000 by exploiting PCA to make feature dimension reduction. Following the same experimental setting of [39] and [52], we randomly select 100 images per category as training data, and regard the remaining samples as test samples. For LLC, the numbers of local bases of LLC ${ }^{*}$ and LLC are set to 30 and 70, respectively, which are the same parameters used in [4] and [39]. Similar to the abovementioned experiments, we also report the mean recognition results (mean \pm std) of our method over ten times run. For fair comparison, we directly cite the results reported in LC-KSVD [39] for performance evaluation. The experimental results are summarized in Table VII. There is no doubt that our approach maintains the highest recognition accuracies and outperforms all the competing methods. Specifically, at least 3\% improvements are achieved when comparing with the other methods.

\section{E. Experimental Analysis}

Based on the numerical experimental results shown in Table I-VII, the following observations are reached.

First, the proposed BDLRR method gains the best performances in comparison with all the compared state-of-the-art methods for recognition tasks on eight data sets. This demonstrates that the proposed method enables to effectively learn a

\footnotetext{
${ }^{4}$ In this experiment, the features used are publicly available at http:// www.umiacs.umd.edu/ zhuolin/projectlcksvd.html.
}

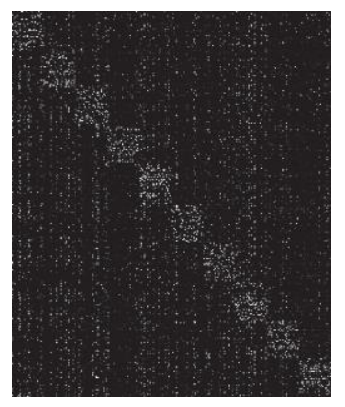

(a)

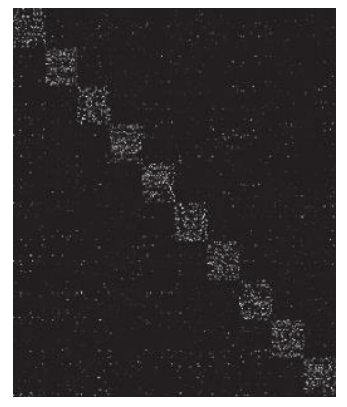

(b)
Fig. 1. Data representation comparisons on the extended YaleB data set. (a) and (b) Data representations of the test set obtained using SRC and BDLRR, respectively. The data representation values are multiplied by five.

discriminative and robust representation from data. Moreover, we can conclude that it is beneficial to image recognition when transferring the original image features to the discriminative BDLRR based on the pivot features, i.e., training features, in a semisupervised manner.

Second, the proposed BDLRR is significantly superior to some related methods, i.e., RPCA, LRSI, LatLRR, LRLR, LRRR, and CBDS, which demonstrates the benefit and necessity of imposing the discriminative structure on LRR and leveraging the $l_{21}$-norm to overcome noise and outliers. With the purpose of constructing the discriminative structure, the margin between block-diagonal and off-block-diagonal components is enlarged such that the incoherent data representation is boosted and the coherent data representation is enhanced simultaneously. Furthermore, it is also revealed that jointly learning the training and test representations can greatly improve the performance of recognition tasks.

Third, an interesting scenario in the experimental results is that there does not exist the absolute best algorithm among all the compared methodologies on eight data sets, because the performance relative to each other is mixed and inconsistent for different recognition applications. However, our BDLRR method outperforms all other methods on these low-resolution, limited training sample experiments. The main reason may be that our method intrinsically inherits the superiorities of sparse, low-rank, structured, and elastic-net representation learning techniques. Specifically, the low-rank regularization, on the one hand, can effectively mine the underlying structure of data correlation, and the global latent structure of the data matrix is uncovered. One the other hand, the sparsity characteristic mainly focuses on finding the nearest subspace of data. However, they neglect the fact that constructing blockdiagonal representation is the most straightforward fashion to explore the intrinsic structure of data and elucidate the nearest subspace of data points. For instance, we use the first ten classes of test samples from the extended YaleB data set to visually present the representation results of SRC and BDLRR, which are shown in Fig. 1. Images of the first ten subjects from the extended YaleB data set are used for experiments. We randomly select 35 images per subject as training samples and treat the rest of images as test samples. All images are rearranged by Assumption 1. From Fig. 1, we can see that our method can more clearly show the nearest 


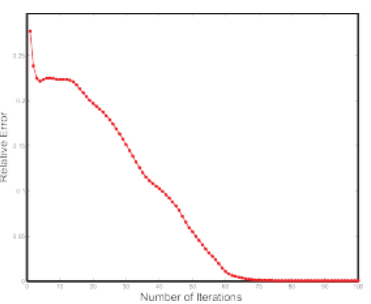

(a)

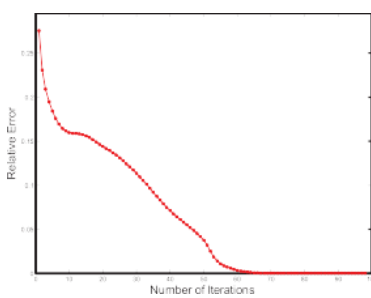

(b)

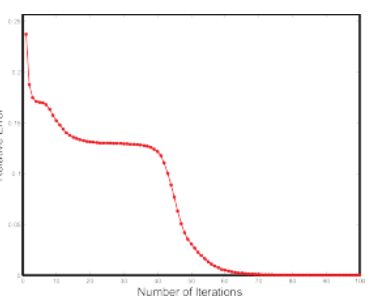

(c)

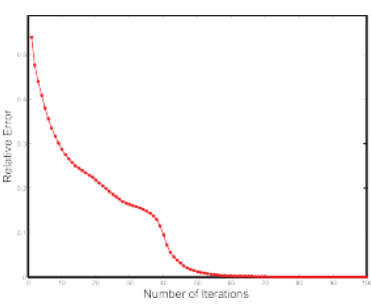

(d)

Fig. 2. Convergence curves of the proposed method on different databases. (a)-(d) Convergence curves on the extended YaleB (\#Tr 35), AR (\#Tr 20), USPS (\#Tr 90), and Char74K-15 (\#Tr 15) data sets, respectively.

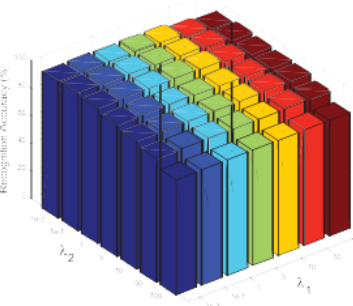

(a)

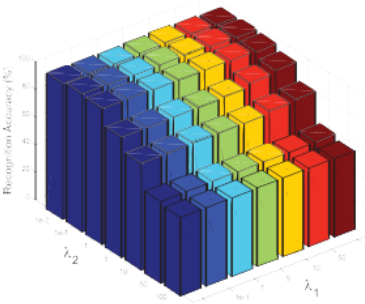

(b)

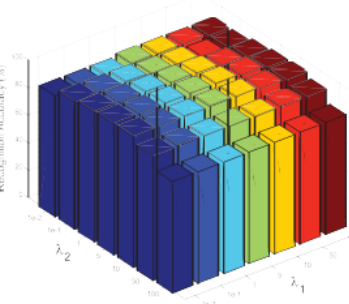

(c)

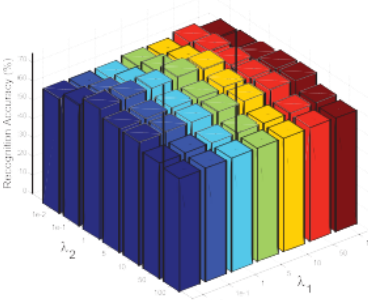

(d)

Fig. 3. Performance evaluation (\%) of BDLRR versus parameters $\lambda_{1}$ and $\lambda_{2}$ on (a) extended YaleB (\#Tr 35), (b) AR (\#Tr 20), (c) USPS (\#Tr 90), and (d) Char74K-15 (\#Tr 15) data sets.

subspace (block-diagonal structure) of test samples, leading to better recognition results.

Fourth, our BDLRR method consistently outperforms CBDS and LatLRR on all data sets. For CBDS, it locally enforces the classwise diagonal structure on the low-rank criterion, whereas our BDLRR method globally imposes the block-diagonal constraint on the low-rank criterion by directly minimizing the off-block-diagonal components. Moreover, our method further enhances the within block-diagonal structure to be more compact by increasing the coherent intraclass representation. For LatLRR, it extracts salient features from observations for recognition, and the unfavorable performance may result from the certain truth that too many image details are lost.

Finally, we can see that the proposed method can overcome the difficulty of noise-induced data uncertainty in face recognition, such as occlusion, disguise, severe illumination changes, and expression variations. Moreover, our method works well on the challenging natural scene character recognition task, which further indicates that BDLRR is robust to the obstacles and difficulties of scene text images, such as complex background, low-resolution, occlusion, blurring, and the changes of text size or font.

\section{F. Convergence and Parameter Sensitiveness Analysis}

In this section, the convergence property of BDLRR and the influence of parameter selection are empirically studied on four data sets, i.e., the extended YaleB, AR, USPS, and Char74K-15 data sets.

1) Convergence Study: The theoretical convergence proof of the proposed optimization method is analyzed in Section IV-C. It is demonstrated that BDLRR can converge to a stationary point under mild conditions. Now we experimentally validate its convergence on different data sets to demonstrate its efficient convergence. The convergence curves on four data sets are presented in Fig. 2, where \#Tr denotes the number of training samples per subject selected for experiments. Similar to [8], the relative error (i.e., $\times X-X_{\text {tr }} Z-E \times_{F} / \times X \times_{F}$ ) is employed to show its convergence. We can see that the relative error generally decreases with the increasing number of iterations. More specifically, although the relative error exists a little vibration at the first 15 iterations on the extended YaleB data set, the overall values of the relative error change only slightly after 60 iterations for these four data sets shown in Fig. 2, which demonstrates that the proposed optimization algorithm holds the convergent nature.

2) Parameter Sensitiveness: In the proposed optimization problem (8), there are three parameters to be tuned. In our experiments, it is observed that the performance of BDLRR is not sensitive to $\lambda_{3}$ when it is in the range of $[10,25]$, which is also an empirical setting. To test how the remaining parameters $\lambda_{1}$ and $\lambda_{2}$ influence the performance of BDLRR, we perform extensive experiments to validate their robustness. Similar to convergence validations, we still use the extended YaleB, AR, USPS, and Char74K-15 data sets for evaluation. Fig. 3 shows the performance variations with respect to parameters $\lambda_{1}$ and $\lambda_{2}$. We can see that the performance of our BDLRR method is generally insensitive to the varying values of $\lambda_{1}$ and $\lambda_{2}$. More specifically, the performance is promising when parameter $\lambda_{1}$ is not too large or small, which indicates the necessity of boosting the extra-class data incoherent representation. Moreover, for parameter $\lambda_{2}$, it is easy to see that it should be small, and the best results are usually achieved when the value is smaller than 1, yet bigger than 0.01 . The possible reason of a smaller $\lambda_{2}$ may be that the Euclidean distance metric used in our experiments is too simple to perfectly measure the similarity of samples. However, we have achieved very impressive experimental 
results, even with a simple distance metric. In a word, our BDLRR method is robust to parameter changes in most cases.

\section{G. Limitation}

From the objective function of our BDLRR method, i.e., (8), we can see that the proposed model is a semisupervised representation learning model and concurrently learns both block-diagonal representations of training and test samples, which indicates that the test samples and the label of training samples are both given in the learning process. However, in some cases, we cannot get access of test data at the training stage, which may limit the generalization of our model. To this end, we extend our BDLRR method to address the out-ofsample problem in Section IV-E to circumvent this problem. In this way, our results are somewhat subject to the learning capability of algorithms in handling the out-of-sample cases. Fortunately, these methods have been examined to effectively formulate favorable representations of new instances. Moreover, the learned data representations of training samples are reasonably block-diagonal in the training stage, which in turn guarantees the satisfactory recognition results.

\section{CONCLUSION}

In this paper, we have proposed a novel discriminative block-diagonal representation learning model, i.e., BDLRR, for robust image recognition. BDLRR focuses on learning a discriminative data representation by imposing an effective structure in an LRR framework, where the extra-class incoherent representation and the intraclass coherent representation are simultaneously enhanced. The proposed method incorporates the learned BDLRR into the semisupervised model to collaboratively optimize the training data representation and test data representation, and then, an efficient linear classifier is obtained to perform final robust image recognition. Moreover, an effective optimization algorithm is developed to solve the resulting optimization problem. Last but not least, the proposed method was evaluated on eight publicly available benchmark data sets for three different recognition tasks. Extensive experimental results have demonstrated that the proposed BDLRR method is superior to the state-of-the-art methods.

\section{ACKNOWLEDGMENT}

The authors would like to thank Prof. Chenglin Liu and Dr. Xuyao Zhang for many inspiring discussions and constructive suggestions. Moreover, they would also like to thank the editor, an associate editor, and referees for helpful comments and suggestions, which greatly improved this paper.

\section{REFERENCES}

[1] Y. Bengio, A. Courville, and P. Vincent, "Representation learning: A review and new perspectives," IEEE Trans. Pattern Anal. Mach. Intell., vol. 35, no. 8, pp. 1798-1828, Aug. 2013.

[2] Z. Zhang, Y. Xu, J. Yang, X. Li, and D. Zhang, "A survey of sparse representation: Algorithms and applications," IEEE Access, vol. 3, no. 1, pp. 490-530, May 2015.

[3] J. Wright, A. Y. Yang, A. Ganesh, S. S. Sastry, and Y. Ma, "Robust face recognition via sparse representation," IEEE Trans. Pattern Anal. Mach. Intell., vol. 31, no. 2, pp. 210-227, Feb. 2009.
[4] J. Wang, J. Yang, K. Yu, F. Lv, T. Huang, and Y. Gong, "Localityconstrained linear coding for image classification," in Proc. IEEE Conf. Comput. Vis. Pattern Recognit., Jun. 2010, pp. 3360-3367.

[5] S. Li and Y. Fu, "Learning balanced and unbalanced graphs via low-rank coding," IEEE Trans. Knowl. Data Eng., vol. 27, no. 5, pp. 1274-1287, May 2015.

[6] G. Liu, Z. Lin, S. Yan, J. Sun, Y. Yu, and Y. Ma, "Robust recovery of subspace structures by low-rank representation," IEEE Trans. Pattern Anal. Mach. Intell., vol. 35, no. 1, pp. 171-184, Jan. 2013.

[7] G. Liu and S. Yan, "Latent low-rank representation for subspace segmentation and feature extraction," in Proc. IEEE Int. Conf. Comput. Vis., Nov. 2011, pp. 1615-1622.

[8] $\mathrm{S}$. $\mathrm{Li}$ and $\mathrm{Y}$. Fu, "Learning robust and discriminative subspace with lowrank constraints," IEEE Trans. Neural Netw. Learn. Syst., vol. 27, no. 11, pp. 2160-2173, Nov. 2016, doi: 10.1109/TNNLS.2015.2464090.

[9] S. Xiao, M. Tan, D. Xu, and Z. Y. Dong, "Robust kernel low-rank representation," IEEE Trans. Neural Netw. Learn. Syst., vol. 27, no. 11, pp. 2268-2281, Nov. 2016.

[10] Y. Zhang, Z. Jiang, and L. S. Davis, "Learning structured low-rank representations for image classification," in Proc. IEEE Conf. Comput. Vis. Pattern Recognit., Jun. 2013, pp. 676-683.

[11] Z. Li, Z. Lai, Y. Xu, J. Yang, and D. Zhang, "A locality-constrained and label embedding dictionary learning algorithm for image classification," IEEE Trans. Neural Netw. Learn. Syst., vol. 28, no. 2, pp. 278-293, Feb. 2017.

[12] Y. Xu, Z. Zhong, J. Yang, J. You, and D. Zhang, "A new discriminative sparse representation method for robust face recognition via $l_{2}$ regularization," IEEE Trans. Neural Netw. Learn. Syst., 2017, doi: 10.1109/TNNLS.2016.2580572.

[13] F. Nie, H. Huang, X. Cai, and C. H. Ding, "Efficient and robust feature selection via joint $l_{2,1}$-norms minimization," in Proc. Adv. Neural Inf. Process. Syst., 2010, pp. 1813-1821.

[14] Y. Xu, D. Zhang, J. Yang, and J.-Y. Yang, "A two-phase test sample sparse representation method for use with face recognition," IEEE Trans. Circuits Syst. Video Technol., vol. 21, no. 9, pp. 1255-1262, Sep. 2011.

[15] C.-Y. Lu, H. Min, J. Gui, L. Zhu, and Y.-K. Lei, "Face recognition via weighted sparse representation," J. Vis. Commun. Image Represent., vol. 24, no. 2, pp. 111-116, 2013.

[16] L. Zhang, M. Yang, and X. Feng, "Sparse representation or collaborative representation: Which helps face recognition?" in Proc. IEEE Int. Conf. Comput. Vis., Nov. 2011, pp. 471-478.

[17] Z. Zhang, L. Wang, Q. Zhu, Z. Liu, and Y. Chen, "Noise modeling and representation based classification methods for face recognition," Neurocomputing, vol. 148, pp. 420-429, Jan. 2015.

[18] I. Naseem, R. Togneri, and M. Bennamoun, "Linear regression for face recognition," IEEE Trans. Pattern Anal. Mach. Intell., vol. 32, no. 11, pp. 2106-2112, Nov. 2010.

[19] Z. Zhang, Z. Lai, Y. Xu, L. Shao, J. Wu, and G.-S. Xie, "Discriminative elastic-net regularized linear regression," IEEE Trans. Image Process., vol. 26, no. 3, pp. 1466-1481, Mar. 2017.

[20] X. Cai, C. Ding, F. Nie, and H. Huang, "On the equivalent of low-rank linear regressions and linear discriminant analysis based regressions," in Proc. 19th ACM SIGKDD Int. Conf. Knowl. Discovery Data Mining, 2013, pp. 1124-1132.

[21] E. J. Candès, X. Li, Y. Ma, and J. Wright, "Robust principal component analysis?" J. ACM, vol. 58, no. 3, p. 11, May 2011.

[22] C.-P. Wei, C.-F. Chen, and Y.-C. F. Wang, "Robust face recognition with structurally incoherent low-rank matrix decomposition," IEEE Trans. Image Process., vol. 23, no. 8, pp. 3294-3307, Aug. 2014.

[23] Y. Li, J. Liu, Z. Li, Y. Zhang, H. Lu, and S. Ma, "Learning lowrank representations with classwise block-diagonal structure for robust face recognition," in Proc. 28th AAAI Conf. Artif. Intell., 2014, pp. 2810-2816

[24] S. Li, M. Shao, and Y. Fu, "Multi-view low-rank analysis for outlier detection," in Proc. SIAM Int. Conf. Data Mining, 2015, pp. $748-756$.

[25] L. Zhuang et al., "Constructing a nonnegative low-rank and sparse graph with data-adaptive features," IEEE Trans. Image Process., vol. 24, no. 11 , pp. 3717-3728, Nov. 2015.

[26] E. Elhamifar and R. Vidal, "Sparse subspace clustering: Algorithm, theory, and applications," IEEE Trans. Pattern Anal. Mach. Intell., vol. 35, no. 11, pp. 2765-2781, Nov. 2013.

[27] Z. Lin, M. Chen, and Y. Ma. (2010). "The augmented lagrange multiplier method for exact recovery of corrupted low-rank matrices." [Online]. Available: https://arxiv.org/abs/1009.5055 
[28] J.-F. Cai, E. J. Candès, and Z. Shen, "A singular value thresholding algorithm for matrix completion," SIAM J. Optim., vol. 20, no. 4, pp. 1956-1982, 2010.

[29] R. Glowinski and P. Le Tallec, Augmented Lagrangian and OperatorSplitting Methods in Nonlinear Mechanics. Philadelphia, PA, USA: SIAM, 1989.

[30] E. Esser, "Applications of Lagrangian-based alternating direction methods and connections to split Bregman," CAM Rep., vol. 9, p. 31, Apr. 2009.

[31] J. Eckstein and D. P. Bertsekas, "On the Douglas-Rachford splitting method and the proximal point algorithm for maximal monotone operators," Math. Program., vol. 55, no. 1, pp. 293-318, 1992.

[32] D. P. Bertsekas, Convex Optimization Theory. Belmont, CA, USA: Athena Scientific, 2009.

[33] L. N. Trefethen and D. Bau, III, Numerical Linear Algebra. Philadelphia, PA, USA: SIAM, 1997.

[34] C.-C. Chang and C.-J. Lin, "LIBSVM: A library for support vector machines," ACM Trans. Intell. Syst. Technol., vol. 2, no. 3, p. 27, 2011.

[35] A. S. Georghiades, P. N. Belhumeur, and D. Kriegman, "From few to many: Illumination cone models for face recognition under variable lighting and pose," IEEE Trans. Pattern Anal. Mach. Intell., vol. 23, no. 6, pp. 643-660, Jun. 2001.

[36] T. Sim, S. Baker, and M. Bsat, "The CMU pose, illumination, and expression (PIE) database," in Proc. 5th IEEE Int. Conf. Autom. Face Gesture Recognit., May 2002, pp. 46-51.

[37] A. M. Martinez and R. Benavente, "The AR face database," CVC Tech. Rep. 24, Jun. 1998.

[38] G. B. Huang, M. Ramesh, T. Berg, and E. Learned-Miller, "Labeled faces in the wild: A database for studying face recognition in unconstrained environments," Univ. Massachusetts, Amherst, MA, USA, Tech. Rep. 07-49, 2007.

[39] Z. Jiang, Z. Lin, and L. S. Davis, "Label consistent K-SVD: Learning a discriminative dictionary for recognition," IEEE Trans. Pattern Anal. Mach. Intell., vol. 35, no. 11, pp. 2651-2664, Nov. 2013.

[40] S.-J. Wang, J. Yang, M.-F. Sun, X.-J. Peng, M.-M. Sun, and C.-G. Zhou, "Sparse tensor discriminant color space for face verification," IEEE Trans. Neural Netw. Learn. Syst., vol. 23, no. 6, pp. 876-888, Jun. 2012.

[41] J. J. Hull, "A database for handwritten text recognition research," IEEE Trans. Pattern Anal. Mach. Intell., vol. 16, no. 5, pp. 550-554, May 1994.

[42] T. E. de Campos, B. R. Babu, and M. Varma, "Character recognition in natural images," in Proc. Int. Conf. Comput. Vis. Theory Appl. (VISAPP), vol. 2. Lisbon, Portugal, 2009, pp. 273-280.

[43] K. Wang, B. Babenko, and S. Belongie, "End-to-end scene text recognition," in Proc. IEEE Int. Conf. Comput. Vis., Barcelona, Spain, Nov. 2011, pp. 1457-1464.

[44] Z. Zhang, Y. Xu, and C.-L. Liu, "Natural scene character recognition using robust PCA and sparse representation," in Proc. IAPR Int. Workshop Document Anal. Syst. (DAS), 2016, pp. 340-345.

[45] S. Tian, S. Lu, B. Su, and C. L. Tan, "Scene text recognition using cooccurrence of histogram of oriented gradients," in Proc. 12th Int. Conf. Document Anal. Recognit., Washington, DC, USA, 2013, pp. 912-916.

[46] B. Su, S. Lu, S. Tian, J. H. Lim, and C. L. Tan, "Character recognition in natural scenes using convolutional co-occurrence HOG," in Proc. 22nd Int. Conf. Pattern Recognit., Istanbul, Turkey, 2014, pp. 2926-2931.

[47] Z. R. Tan, S. Tian, and C. L. Tan, "Using pyramid of histogram of oriented gradients on natural scene text recognition," in Proc. Int. Conf. Image Process., Paris, France, 2014, pp. 2629-2633.

[48] C.-Y. Lee, A. Bhardwaj, W. Di, V. Jagadeesh, and R. Piramuthu, "Region-based discriminative feature pooling for scene text recognition," in Proc. IEEE Conf. Comput. Vis. Pattern Recognit., Columbus, OH, USA, Jun. 2014, pp. 4050-4057.

[49] T. Q. Phan, P. Shivakumara, S. Tian, and C. L. Tan, "Recognizing text with perspective distortion in natural scenes," in Proc. 14th IEEE Int Conf. Comput. Vis., Sydney, NSW, Australia, Dec. 2013, pp. 569-576.

[50] C. Yi, X. Yang, and Y. Tian, "Feature representations for scene text character recognition: A comparative study," in Proc. 12th Int. Conf. Document Anal. Recognit., Washington, DC, USA, 2013, pp. 907-911.

[51] C. Yi and Y. Tian, "Scene text recognition in mobile applications by character descriptor and structure configuration," IEEE Trans. Image Process., vol. 23, no. 7, pp. 2972-2982, Jul. 2014

[52] S. Lazebnik, C. Schmid, and J. Ponce, "Beyond bags of features: Spatial pyramid matching for recognizing natural scene categories," in Proc. IEEE Conf. Comput. Vis. Pattern Recognit., Jun. 2006, pp. 2169-2178.

[53] X.-C. Lian, Z. Li, B.-L. Lu, and L. Zhang, "Max-margin dictionary learning for multiclass image categorization," in Proc. Eur. Conf. Comput. Vis., 2010, pp. 157-170.
[54] J. Yang, K. Yu, Y. Gong, and T. Huang, "Linear spatial pyramid matching using sparse coding for image classification," in Proc. IEEE Conf. Comput. Vis. Pattern Recognit., Jun. 2009, pp. 1794-1801.

[55] Y.-L. Boureau, F. Bach, Y. LeCun, and J. Ponce, "Learning mid-level features for recognition," in Proc. IEEE Conf. Comput. Vis. Pattern Recognit., Jun. 2010, pp. 2559-2566.

[56] S. Gao, I. W.-H. Tsang, L.-T. Chia, and P. Zhao, "Local features are not lonely_Laplacian sparse coding for image classification," in Proc. IEEE Conf. Comput. Vis. Pattern Recognit., Jun. 2010, pp. 3555-3561.

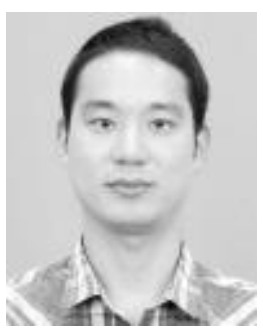

Zheng Zhang received the B.S. degree from the Henan University of Science and Technology, Luoyang, China, in 2012, and the M.S. degree from the Shenzhen Graduate School, Harbin Institute of Technology, Harbin, China, in 2014, where he is currently pursuing the Ph.D. degree in computer science and technology.

His current research interests include pattern recognition, machine learning, and computer vision.

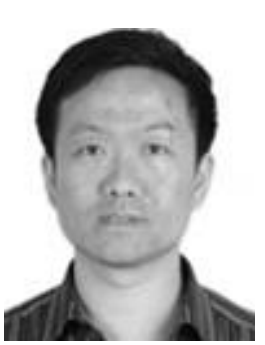

Yong Xu (M'06-SM'15) was born in Sichuan, China, in 1972. He received the B.S. degree and M.S. degree from the Air Force Institute of Meteorology, Beijing, China, in 1994 and 1997, respectively, and the Ph.D. degree in pattern recognition and intelligence system from the Nanjing University of Science and Technology, Nanjing, China, in 2005 .

$\mathrm{He}$ is currently with the Shenzhen Graduate School, Harbin Institute of Technology, Harbin, China. His current interests include pattern recognition, biometrics, machine learning, and video analysis.

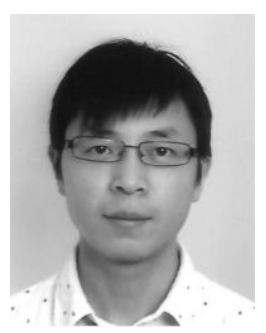

Ling Shao (M'09-SM'10) was a Senior Scientist with Philips Research, Eindhoven, The Netherlands, from 2005 to 2009. From 2009 to 2014, he was a Senior Lecturer with The University of Sheffield, Sheffield, U.K. From 2014 to 2016, he was a Professor with Northumbria University, Newcastle upon Tyne, U.K. He is currently a Professor with the School of Computing Sciences, University of East Anglia, Norwich, U.K. His current research interests include computer vision, image/video processing, and machine learning.

Dr. Shao is a fellow of the British Computer Society and the Institution of Engineering and Technology. He is an Associate Editor of the IEEE Transactions on Image Processing, the IEEE Transactions on NeURAL NETWORKS AND LEARNING Systems, and several other journals.

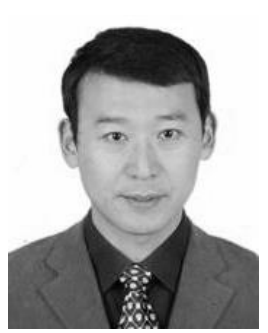

Jian Yang received the B.S. degree in mathematics from Jiangsu Normal University, Xuzhou, China, in 1995; the M.S. degree in applied mathematics from Changsha Railway University, Changsha, China, in 1998; and the Ph.D. degree in pattern recognition and intelligence systems from the Nanjing University of Science and Technology (NUST), Nanjing, China, in 2002.

In 2003, he was a Post-Doctoral Researcher with the University of Zaragoza, Zaragoza, Spain. From 2004 to 2006, he was a Post-Doctoral Fellow with the Biometrics Center, The Hong Kong Polytechnic University, Hong Kong. From 2006 to 2007, he was a Post-Doctoral Fellow with the Department of Computer Science, New Jersey Institute of Technology, Newark, NJ, USA. $\mathrm{He}$ is currently a Professor with the School of Computer Science and Technology, NUST. He has authored over 80 scientific papers in pattern recognition and computer vision. His journal papers have been cited over 1600 times in the ISI Web of Science, and 2800 times in the Web of Scholar Google. His current research interests include pattern recognition, computer vision, and machine learning.

Dr. Yang is an Associate Editor of the Pattern Recognition Letters and the IEEE Transaction on Neural Networks and Learning Systems. 